\title{
An Efficient Approach to the Modeling of Compressive Transverse Cracking in Composite Laminates
}

W. Steenstra 



\title{
AN EFFICIENT APPROACH TO THE MODELING OF Compressive Transyerse Cracking IN COMPOSITE LAMINATES
}

by

\section{W. Steenstra}

in partial fulfillment of the requirements for the degree of

\author{
Master of Science \\ in Civil Engineering \\ at the Delft University of Technology, \\ to be defended publicly on Thursday January 8, 2015 at 3:45 PM.
}

Student number: 1361481

Thesis committee: Prof. dr. ir. L. J. Sluys, Dr. ir. F. P. van der Meer, Dr. ir. S. R. Turteltaub, Ir. L. J. M. Houben,
Computational Mechanics (CiTG), TU Delft Computational Mechanics (CiTG), TU Delft Aerospace Structures \& Materials (A\&E), TU Delft Road and Railway Engineering (CiTG), TU Delft 



\section{PREFACE}

About seven months ago, I was introduced to computational modeling of composite laminates. At that time, a poster that presented the work of Bert Sluys and Frans van der Meer caught my eye. I did not realize that this was going to be the basis of my master thesis! I'm very thankful that I've had to chance to work with Bert and Frans and that they have guided me through my final project. After a course in C++ and Jem Jive and a lot of hard work, the composite laminate failure model resulted that I describe in this thesis. To accompany it, I wrote a paper summarizing my findings, something I'm very excited about. As challenging as the computational modeling of composite laminates was, being able to explain what I spent my days doing to my friends and family was equally as difficult. I hope with this paper and my presentation I can make it clear to them.

I want to thank my graduation committee for guiding me through the process of a thesis and introducing me to the fascinating world of composite laminates.

I also want to thank my friends and family for their support and their coffee cards.

I hope you enjoy reading this thesis as much as I enjoyed writing it.

W. Steenstra

Delft, January 2015 



\section{Contents}

1 Introduction 1

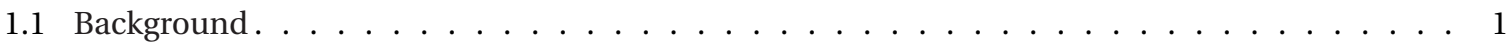

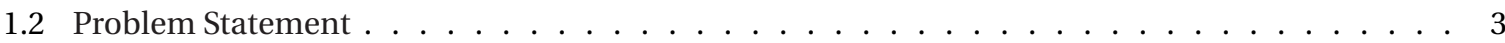

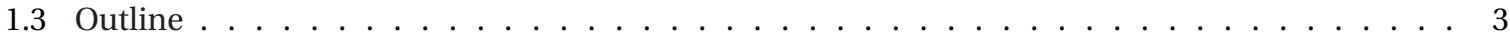

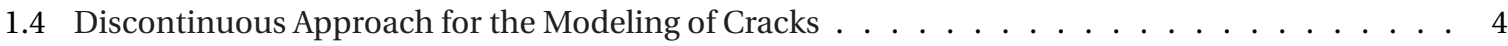

1.4 .1 Cohesive Law . . . . . . . . . . . . . . . . . . . . . . . 4

1.4 .2 Interface Elements. . . . . . . . . . . . . . . . . . . . 5

1.4.3 The Phantom Node Method . . . . . . . . . . . . . . . . . . . . . . . 6

2 Reference Model 9

2.1 Large Displacements . . . . . . . . . . . . . . . . . . . . . . . . . . . . . . . 9

2.2 Material Properties . . . . . . . . . . . . . . . . . . . . . . . . 10

2.3 Buckling Length. . . . . . . . . . . . . . . . . . . . . . . . . . . . . 14

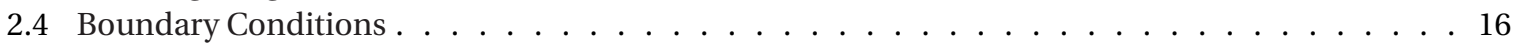

3 Transformed Interface Model $\quad 17$

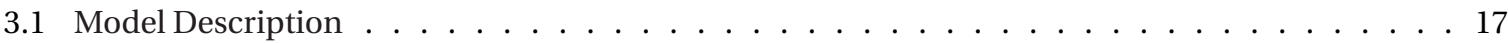

3.2 Single Ply . . . . . . . . . . . . . . . . . . . . . . . . . . 20

3.3 Laminate Analysis. . . . . . . . . . . . . . . . . . . . . . . . . . . . 21

3.3.1 Mesh Refinement . . . . . . . . . . . . . . . . . . . . . . . . . . 22

3.3.2 Tractions in Interface Elements . . . . . . . . . . . . . . . . . . . 23

3.3 .3 Ply Thickness Ratio . . . . . . . . . . . . . . . . . . . . . . . . . . 26

3.3 .4 Conclusion. . . . . . . . . . . . . . . . . . . . . . . 27

4 3D Analysis 29

4.1 Model Description . . . . . . . . . . . . . . . . . . . . . . . . . . . 29

4.2 Laminate Analysis. . . . . . . . . . . . . . . . . . . . . . . . . . 32

4.2 .1 Mesh Refinement . . . . . . . . . . . . . . . . . . . . . . 33

4.2 .2 Fiber Direction. . . . . . . . . . . . . . . . . . . . . . . . . . . 33

4.2 .3 Fracture Plane Angle . . . . . . . . . . . . . . . . . . . . . . . . . . . . . 36

5 Conclusion and Recommendations $\quad 37$

$\begin{array}{ll}\text { Bibliography } & 39\end{array}$ 



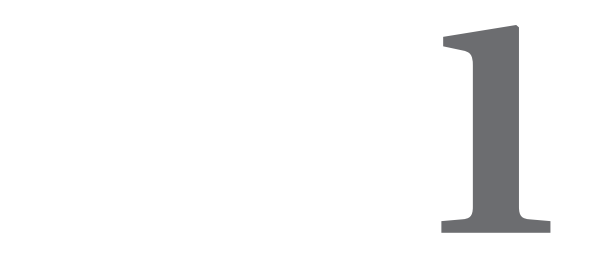

INTRODUCTION

\subsection{Background}

Composite laminates are widely applied as a structural material (e.g. in aircraft industries). Since the prediction of the reliability of composite structures is difficult, the use of these materials leads to high safety factors and/or extensive testing. A typical large airframe, for example, requires $\sim 10^{4}$ tests to achieve safety certification [1]. Therefore, research is still ongoing to predict failure of composite laminates using numerical models.

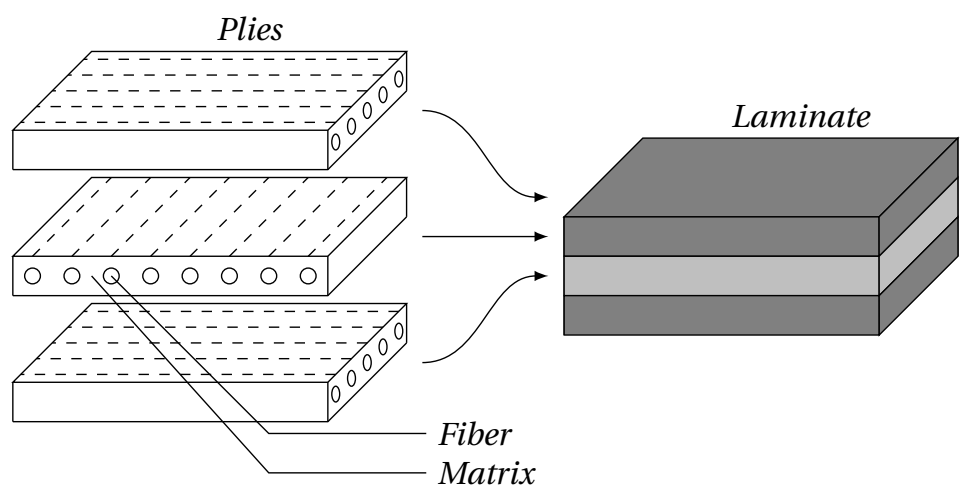

Figure 1.1: Layup of a composite laminate. Microlevel (left) and mesolevel (right).

The main difficulty in modeling composite laminate failure is the different failure processes that may occur due to the configuration of the high performance material. As shown in Figure 1.1, a laminate is a layered material, and composite in this case means that each layer in turn is composed of two materials: fibers (e.g. glass or carbon) and matrix material (e.g. epoxy). Failure processes that are typically distinguished are fiber rupture, fiber kinking, matrix cracking, fiber-matrix debonding and delamination. Even though the individual processes occur on a small scale, a combination can eventually lead to ultimate failure of the structure. See for instance Figure 1.2, where inter-fiber failure in the mid ply is followed by delamination and local buckling of the outer plies.

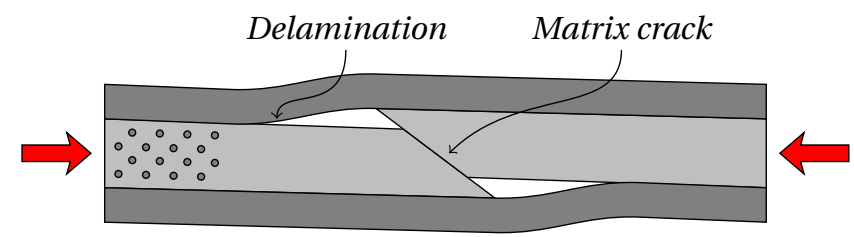

Figure 1.2: Schematic illustration of transverse compressive fracture causing delamination and local buckling. 


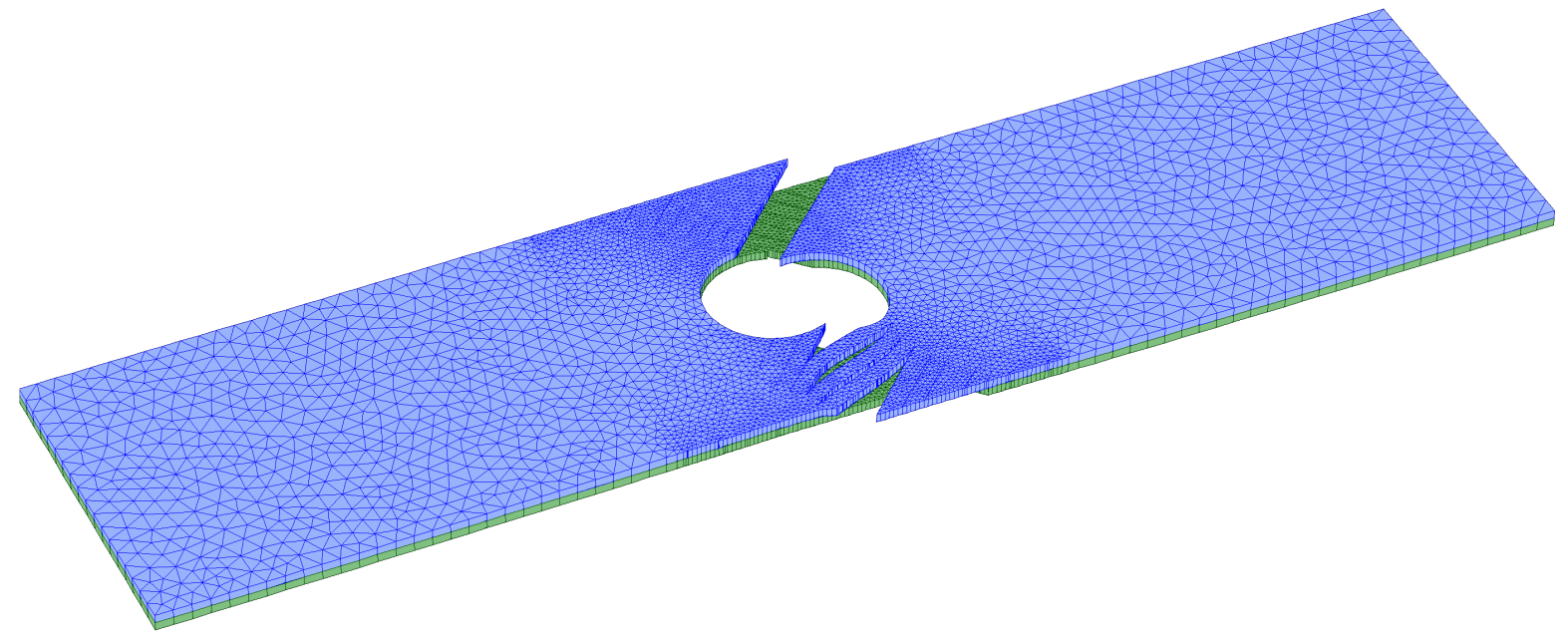

Figure 1.3: Deformed mesh from open hole analysis by Van der Meer's 3D model. Multiple cracks per ply are modeled using XFEM.

Recent work by Van der Meer has resulted in a computational model for analysis of laminate failure [2]. The scale of observation of this model is the mesolevel. Plies are modeled separately, using a single layer of elements per ply. This way, a distinction is easily made between delamination and ply failure. Delamination is taken into account, using interlaminar interface elements as described by Turon [3]. Since Van Der Meer and Sluys showed that a continuum damage model does not always lead to a correct representation of interfiber cracks [4], a discontinuous approach is preferred to model ply failure. In a discontinuous model, matrix cracks can be represented by predefined interface elements. The crack topology and propagation then has to be incorporated in the mesh. This results in laborious meshing when several laminate configurations are analyzed, especially if multiple cracks per ply are considered in 3D (see for instance Figure 1.3). Therefore, a better option is to use the eXtended Finite Element Method (XFEM) [5]. With this method, cracks can occur at arbitrary locations and the direction of the crack is independent of the mesh. The phantom node method is a variation on XFEM. The discontinuous approaches are described in section 1.4 and used throughout this work.

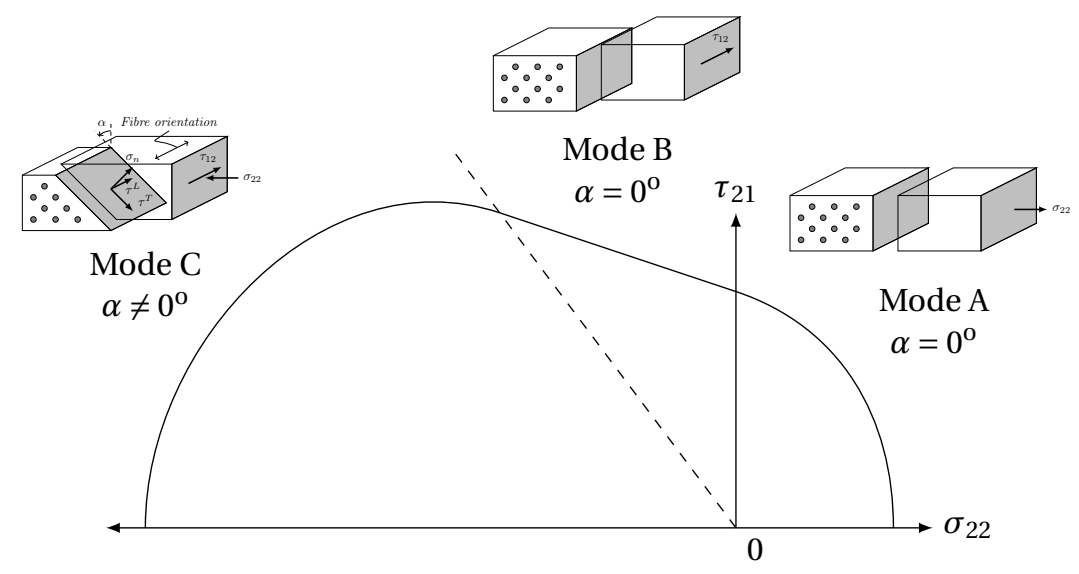

Figure 1.4: Fracture envelope for combined $\left(\sigma_{22}, \tau_{21}\right)$ stresses with fracture modes A, B, C and fracture angle $\alpha$.

Since ply failure in compression differs significantly from ply failure in tension, Van Der Meer's model is limited to tensile load cases. As shown in Figure 1.4, inter-fiber failure can occur in three different modes. When a tensile stress acts on the laminate $\left(\sigma_{22}>0\right)$, Mode A occurs with a fracture plane $\alpha$ perpendicular to the mid plane of the ply $(\alpha=0)$. In compression load cases with a relatively high shear force $\tau_{21}$, mode $\mathrm{B}$ occurs where the fracture plane is still perpendicular to the mid plane of the ply $\left(\sigma_{22}<0, \alpha=0\right)$. For compressive load cases with a relatively high $\sigma_{22} / \tau_{21}$-ratio, mode $\mathrm{C}$ occurs where the fractue plane is inclined with respect to the mid plane of the ply $\left(\sigma_{22}<0, \alpha \neq 0\right)$. Puck and Schürmann found that for pure compressive loading $\left(\sigma_{22}<0, \tau_{21}=0\right)$, the fracture plane tends to be inclined with respect to the load direction with an 
angle $\alpha_{0}=53^{\circ}[6]$.

For Van Der Meer's model to be good enough for predictive simulation of the complete failure process in laminates, the compressive behavior has to be included. Besides the before-mentioned disadvantages of interface elements, predefining the fracture plane with interface elements would mean that the failure mode has to be known in advance. It is possible to account for the angle of the fracture plane explicitly with XFEM. However, in 3D, the complexity of the algorithms that deal with the intersection of the finite elements by the crack plane increases significantly when the crack plane is no longer perpendicular to the mid-plane of the ply. Therefore, an efficient approach is needed to model compressive failure of composite laminates using a vertical crack plane. When the crack topology remains vertical, an inclined crack can only be approximated by a modification in the constitutive law. For the discontinuous approaches used in Van Der Meer's model, this constitutive law is a cohesive law related to the discontinuous displacement field.

\subsection{Problem Statement}

The difficulty of including compressive behavior in a complete laminate failure model, as described in the previous section leads to the following problem statement:

\section{Can the compressive behavior of composite laminate failure be approximated by changing the cohesive law instead of the crack topology?}

The idea is to use a transformation in the cohesive law such that vertical mesh elements act as if the fracture plane has an inclination. Interpenetration of neighboring elements is typically prevented by stiffness recovery for compressive loading in the normal direction of an interface. With a transformation of the local reference frame just before the cohesive law is applied, stiffness recovery will be applied along an inclined axis. As a result, elements near the crack path will slide over each other when a certain failure criterion is reached (see Figure 1.5, below).

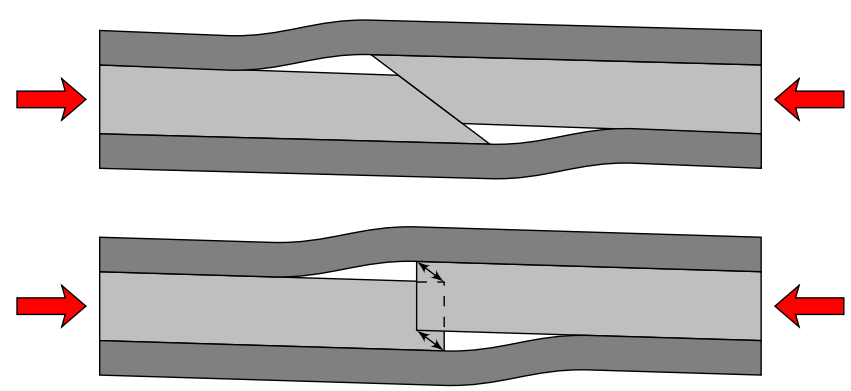

Figure 1.5: Schematic illustration of compressive failure (above) and the approximation of it, using a vertical crack plane (below)

\subsection{Outline}

Using the model for full-laminate tensile failure as a framework, several extensions need to be implemented. In order to keep the model clear and simple, the idea is to work step by step towards a final failure model for compressively loaded laminates. In the same chronological order, the model description and results are presented in this thesis.

To approximate compressive failure using a transformation in the cohesive law, a reference model is needed for verification. In the reference model, predefined interface elements are used to simulate the inclined fracture plane. This so called 'inclined interface model' is introduced in Chapter 2. The need for large displacement, material properties and limitations to the model are discussed.

In order to simulate the inclined fracture plane using vertical elements, a transformation to the cohesive law needs to e applied. In 2D, this is done with vertical interface elements. The so called 'transformed interface model' is introduced in Chapter 3. The method of transformation in the cohesive law is explained and first verified for a compressively loaded single ply. Second, the effect of the transformation on modeling transverse compressive matrix cracking followed by delamination is assessed with the analysis of a $[0 / \overline{90}]$ laminate.

Finally, a three dimensional stress state is analyzed in Chapter 4 . The direction of the crack propagation is set according to the fiber orientation in the cracked ply using the phantom node method. The transformation 
is applied to the cohesive law related to the segments introduced by the phantom node method. The influence of a varying fiber orientation and a varying fracture plane angle, depending on the stress state at onset of fracture, are discussed in more detail.

\subsection{Discontinuous Approach for the Modeling of Cracks}

In a discontinuous approach, cracks are modeled by inserting a discontinuity in the displacement field. Besides the constitutive law for the continuum, an extra constitutive law has to be introduced to apply cohesive forces on the crack surface (Barenblatt [7]). This cohesive law is described in section 1.4.1 for a relatively simple uncoupled model. Section 1.4.2 describes the methodology of interface elements in 2D. The phantom node method is explained in section 1.4.3 for a 3D situation.

\subsubsection{Cohesive Law}

The cohesive law relates the cohesive traction $\mathbf{t}$, applied to the crack surface, to the size of the displacement jump j:

$$
\mathbf{t}=\mathbf{t}(\mathbf{j})
$$

The cohesive law ensures that elements initially approximate rigid behavior until a certain failure criterion is reached. This failure criterion is obtained in a local reference system. After onset of fracture, the relative displacement is the vectorial sum of a traction/compression mode I and a sliding mode II. For pure tension/compression and pure shear, the constitutive relationships are shown in Figure 1.6. The subscribts $o$ and $f$ indicate onset and failure.
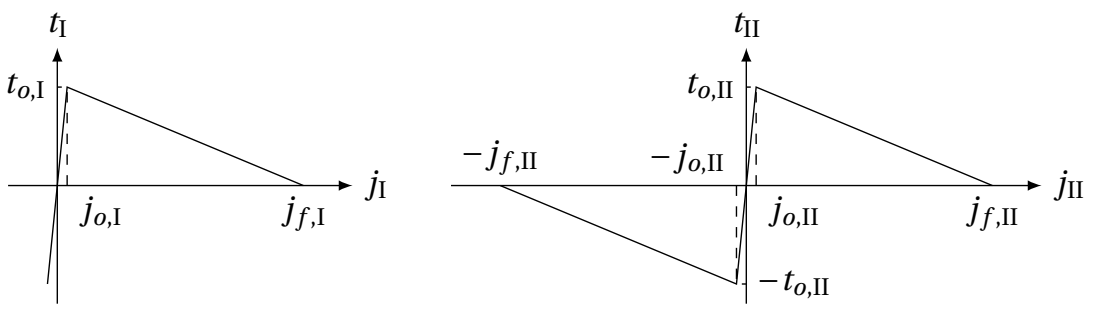

Figure 1.6: Constitutive relationships for pure mode I (left) and pure mode II (right).

For this uncoupled model, the analytical expressions of the cohesive law for mode $i$ are bilinear, onedimensional relationships as described by Alfano [8]. Using the notation $s_{i}[x]$ for mode $i$ :

$$
s_{i}[x]= \begin{cases}\langle x\rangle & \text { if } i=1 \\ |x| & \text { if } i=2\end{cases}
$$

where $\langle x\rangle=\frac{1}{2}(x+|x|)$

$$
t_{i}=\left\{\begin{array}{cc}
K_{i} j_{i} & \text { if }\left(s_{i}\left[j_{i}\right]\right)_{\max } \leq j_{o, i} \text { or } \\
\left(i=\mathrm{I} \text { and } j_{i}<0\right) \\
K_{i} j_{i}\left[1-\left(\frac{\left(s_{i}\left[j_{i}\right]\right)_{\max }-j_{o, i}}{\left(s_{i}\left[j_{i}\right]\right)_{\max }}\right)\left(\frac{j_{f, i}}{j_{f, i}-j_{o, i}}\right)\right] & \text { if } j_{o, i}<\left(s_{i}\left[j_{i}\right]\right)_{\max }<j_{f, i} \text { and } \\
0 & \left(i \neq \mathrm{I} \text { or } j_{i} \geq 0\right) \\
& \text { if }\left(s_{i}\left[j_{i}\right]\right)_{\max } \geq j_{f, i} \text { and } \\
& \left(i \neq \mathrm{I} \text { or } j_{i} \geq 0\right)
\end{array}\right.
$$

where $\left(s_{i}\left[j_{i}\right]\right)_{\max }$ is the maximum value attained by (1.2) to take into account the irreversibility of the interface damage. $K_{i}$ is a dummy stiffness to approximate initially rigid behavior. Figure 1.6 and equation (1.3) show that interpenetration is limited to a certain tolerance depending on the dummy stiffness $K_{\mathrm{I}}$.

When the interaction of two modes is taken into account, a mixed-mode damage law by Turon can be used [3]. Van der Meer formulated a phantom node formulation with the mixed-mode cohesive law [9]. 


\subsubsection{Interface Elements}

Using duplicated nodes along the crack path, the discontinuity in the displacement field can be modeled with interface elements. The nodes of an interface element initially coincide, but they may be driven apart mechanically as shown in Figure 1.7. The displacement jump is defined as the difference between the displacement fields of the two lines.

$$
\mathbf{j}=\mathbf{N a}
$$

With the shape function matrix

$$
\mathbf{N}=\left[\begin{array}{cccccccccc}
N_{1} & 0 & \ldots & N_{n} & 0 & -N_{1} & 0 & \ldots & -N_{n} & 0 \\
0 & N_{1} & \ldots & 0 & N_{n} & 0 & -N_{1} & \ldots & 0 & -N_{n}
\end{array}\right]
$$

and

$$
\mathbf{a}^{\mathrm{T}}=\left\{a_{1 x}, a_{1 y}, \ldots, a_{2 n x}, a_{2 n y}\right\}
$$

Where $n$ is the number of nodes per element and $\mathbf{a}_{i}$ the displacements of node $i$.

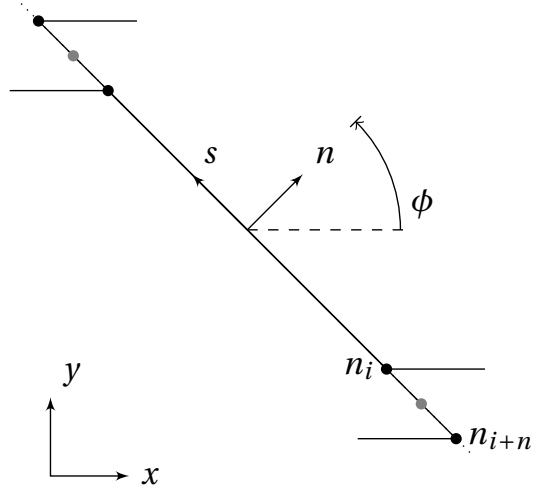

Figure 1.7: Interface element with local $\{n, s\}$-frame in a global $\{x, y\}$-frame.

The displacement jump $\mathbf{j}$ in the local coordinate system is transformed to $\overline{\mathbf{j}}$ in the local $\{n, s\}$-frame system within a standard finite element program. For every integration point, this is done by the rotation matrix $\mathbf{R}_{\phi}$ that maps the global coordinates to the local coordinates. In 2D, it holds:

$$
\mathbf{R}_{\phi} \mathbf{j}=\left[\begin{array}{cc}
\cos (\phi) & \sin (\phi) \\
-\sin (\phi) & \cos (\phi)
\end{array}\right]\left[\begin{array}{c}
j_{x} \\
j_{y}
\end{array}\right]=\left[\begin{array}{c}
j_{n} \\
j_{s}
\end{array}\right]=\overline{\mathbf{j}}
$$

In the local coordinate system, the cohesive law (1.1) is applied to obtain the traction vector $\overline{\mathbf{t}}$ and its linearization with respect to the displacement jump $\overline{\mathbf{T}}=\delta \overline{\mathbf{t}} / \delta \overline{\mathbf{j}} . \overline{\mathbf{t}}$ and $\overline{\mathbf{T}}$ are transformed back into the global $\{x, y\}$ coordinate system with the transposed rotation matrix $\mathbf{R}_{\phi}^{\mathrm{T}}$ :

$$
\begin{gathered}
\mathbf{R}_{\phi}^{\mathrm{T}} \overline{\mathbf{t}}=\left[\begin{array}{cc}
\cos (\phi) & -\sin (\phi) \\
\sin (\phi) & \cos (\phi)
\end{array}\right]\left[\begin{array}{c}
t_{n} \\
t_{s}
\end{array}\right]=\left[\begin{array}{l}
t_{x} \\
t_{y}
\end{array}\right]=\mathbf{t} \\
\mathbf{R}_{\phi}^{\mathrm{T}} \overline{\mathbf{T}} \mathbf{R}_{\phi}=\left[\begin{array}{cc}
\cos (\phi) & -\sin (\phi) \\
\sin (\phi) & \cos (\phi)
\end{array}\right] \overline{\mathbf{T}}\left[\begin{array}{cc}
\cos (\phi) & \sin (\phi) \\
-\sin (\phi) & \cos (\phi)
\end{array}\right]=\mathbf{T}
\end{gathered}
$$

The element force vector $\mathbf{f}_{\text {elem }}$ and the element stiffness matrix $\mathbf{K}_{\text {elem }}$ are then defined by an integral over the interface surface $\Gamma_{i}$ 


$$
\begin{aligned}
\mathbf{f}_{\text {elem }} & =\int_{\Gamma_{i}} \mathbf{N}^{\mathrm{T}} \mathbf{t} d \Gamma \\
\mathbf{K}_{\text {elem }} & =\int_{\Gamma_{i}} \mathbf{N}^{\mathrm{T}} \mathbf{T N} d \Gamma
\end{aligned}
$$

Interface elements are an effective solution to model cracks in relatively simple cases. However, since the discontinuity is defined between elements, this method is not very flexible. For different material configurations, different crack paths need to be predefined in the mesh. Especially in a 3D situation, this can result in laborious meshing.

\subsubsection{The Phantom Node Method}

With the phantom node version of XFEM, cohesive cracking can be modeled with partially active overlapping elements with independent displacement fields ([10], [11] and [12]). The connectivity and active parts of two overlapping elements are shown in Figure 1.8.
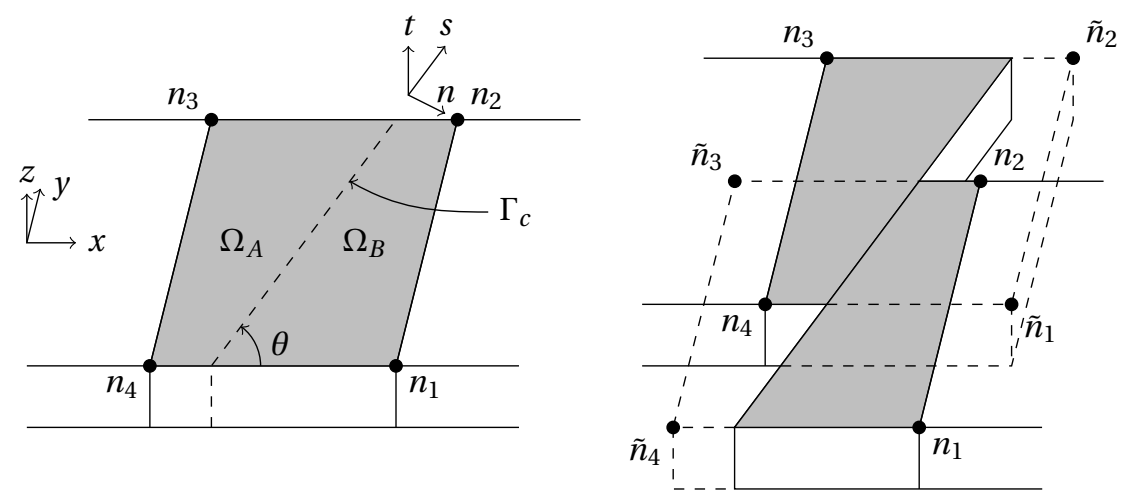

Figure 1.8: Connectivity and active parts of two overlapping elements in phantom node method.

Due to the micro structure of an unidirectional composite laminate, the crack in a ply will propagate in the direction of the fiber orientation. This is taken into account by specifying the angle $\theta$. The displacement jump j over the crack is defined as the difference between the displacement fields of the two initially overlapping elements $A$ and $B$. The displacement jump is transformed to an orthonormal frame that is aligned with the crack by the rotation matrix $\mathbf{R}_{\theta}$. With the $t$-axis parallel to the $z$-axis, the transformation from the displacement jump in the local $\{n, s, t\}$-frame is related to the displacement jump in the global $\{x, y, z\}$-frame by

$$
\mathbf{R}_{\theta} \mathbf{j}=\left[\begin{array}{ccc}
\sin (\theta) & -\cos (\theta) & 0 \\
\cos (\theta) & \sin (\theta) & 0 \\
0 & 0 & 1
\end{array}\right]\left[\begin{array}{c}
j_{x} \\
j_{y} \\
j_{z}
\end{array}\right]=\left[\begin{array}{c}
j_{n} \\
j_{s} \\
j_{t}
\end{array}\right]=\overline{\mathbf{j}}
$$

In the local coordinate system, the cohesive law (1.1) is applied to obtain the traction vector $\overline{\mathbf{t}}$ and its linearization with respect to the displacement jump $\overline{\mathbf{T}}=\delta \overline{\mathbf{t}} / \delta \overline{\mathbf{j}} . \overline{\mathbf{t}}$ and $\overline{\mathbf{T}}$ are transformed back into the global $\{x, y, z\}$ coordinate system with the transposed rotation matrix $\mathbf{R}_{\theta}^{\mathrm{T}}$ :

$$
\begin{gathered}
\mathbf{R}_{\theta}^{\mathrm{T}} \overline{\mathbf{t}}=\mathbf{t} \\
\mathbf{R}_{\theta}^{\mathrm{T}} \overline{\mathbf{T}} \mathbf{R}_{\theta}=\mathbf{T}
\end{gathered}
$$

With the standard finite element shape functions $\mathbf{N}(\mathbf{x})$ and the strain nodal displacement matrix $\mathbf{B}$, the contribution to the internal force vector on the degrees of freedom corresponding with element $A$ and $B$ are defined as 


$$
\mathbf{f}_{A}^{\text {int }}=\int_{\Omega_{A}} \mathbf{B}^{\mathrm{T}} \boldsymbol{\sigma} d \Omega+\int_{\Gamma_{c}} \mathbf{N t} d \Gamma
$$

and

$$
\mathbf{f}_{B}^{\mathrm{int}}=\int_{\Omega_{B}} \mathbf{B}^{\mathrm{T}} \boldsymbol{\sigma} d \Omega-\int_{\Gamma_{c}} \mathbf{N t} d \Gamma
$$

The contribution to the global tangent matrix is

$$
\mathbf{K}=\left[\begin{array}{cc}
\mathbf{K}_{A} & \mathbf{0} \\
\mathbf{0} & \mathbf{K}_{B}
\end{array}\right]+\left[\begin{array}{cc}
\mathbf{K}_{\mathbf{j}} & -\mathbf{K}_{\mathbf{j}} \\
-\mathbf{K}_{\mathbf{j}} & \mathbf{K}_{\mathbf{j}}
\end{array}\right]
$$

with

$$
\begin{gathered}
\mathbf{K}_{i}=\int_{\Omega_{i}} \mathbf{B}^{\mathrm{T}} \mathbf{D B} d \Omega, i=A, B \\
\mathbf{K}_{\mathbf{j}}=\int_{\Gamma_{c}} \mathbf{N}^{\mathrm{T}} \mathbf{T N} d \Gamma
\end{gathered}
$$

where $\mathbf{D}=\partial \boldsymbol{\sigma} / \partial \boldsymbol{\epsilon}$. 



\section{2}

\section{REFERENCE MODEL}

In this Chapter, a model is introduced where the compressive crack is modeled with predefined interface elements. The so called 'inclined interface model' is shown in Figure 2.1. The model is discussed in more detail in the following sections. It will serve as a reference model in a later stage, for a similar model where the fracture plane angle $\alpha$ is included as a transformation in the cohesive law of vertical interface elements. The need for including large displacements is shown in section 2.1. Material properties are discussed in section 2.2. Limitations to the model regarding length/thickness are discussed in section 2.3 for buckling and the influence of the boundary conditions in section 2.4.

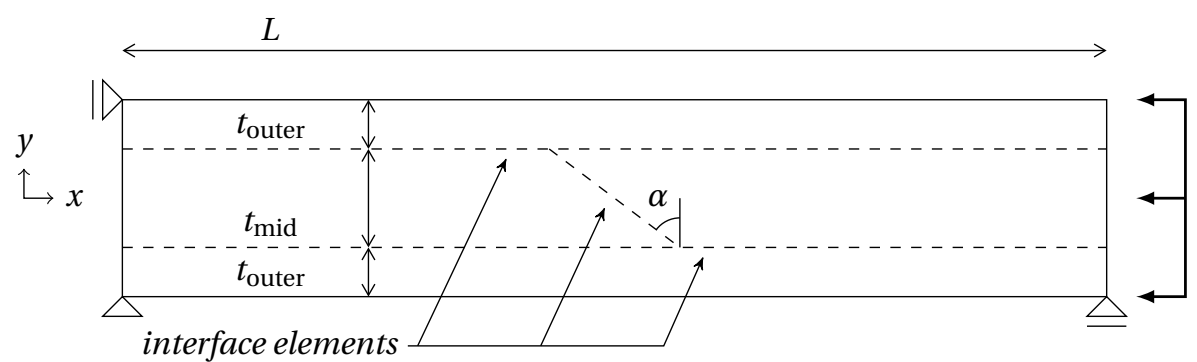

Figure 2.1: Schematic representation of modeling inclined compressive crack in a two-sided clamped $[0 / \overline{90}]_{s}$ laminate using interface elements.

\subsection{Large Displacements}

In the considered case, delamination is followed by local buckling of the outer plies. Therefore, the assumption of small displacements is not valid. For the bulk material, the Green-Lagrange strain tensor is used:

$$
\boldsymbol{\gamma}=\frac{1}{2}\left(\mathbf{F}^{\mathrm{T}} \mathbf{F}-\mathbf{I}\right)
$$

With $\mathbf{I}$, the identity matrix and $\mathbf{F}$, the deformation gradient tensor that relates the deformed configuration $\left(x_{1}, x_{2}\right)$ to the undeformed configuration $\left(\xi_{1}, \xi_{2}\right)$ :

$$
\mathbf{F}=\frac{\partial \mathbf{x}}{\partial \boldsymbol{\xi}}
$$

The deformed configuration of the interface elements is limited to a rotation of the element. In $2 \mathrm{D}$, the rotation of the normal vector of a line is relatively simple while in $3 \mathrm{D}$ the interface element being a plane makes rotation more difficult. As Figure 2.2 shows, taking into account only large displacements in the bulk material already results in the desired snapback behavior. When large displacements are taken into account in the interface elements as well, the load-displacement diagram shows the same snapback behavior, though convergence is still reached when the crack starts to open. In the remainder of this thesis, large displacements are assumed in the bulk material while small rotations are assumed in the interface elements. 


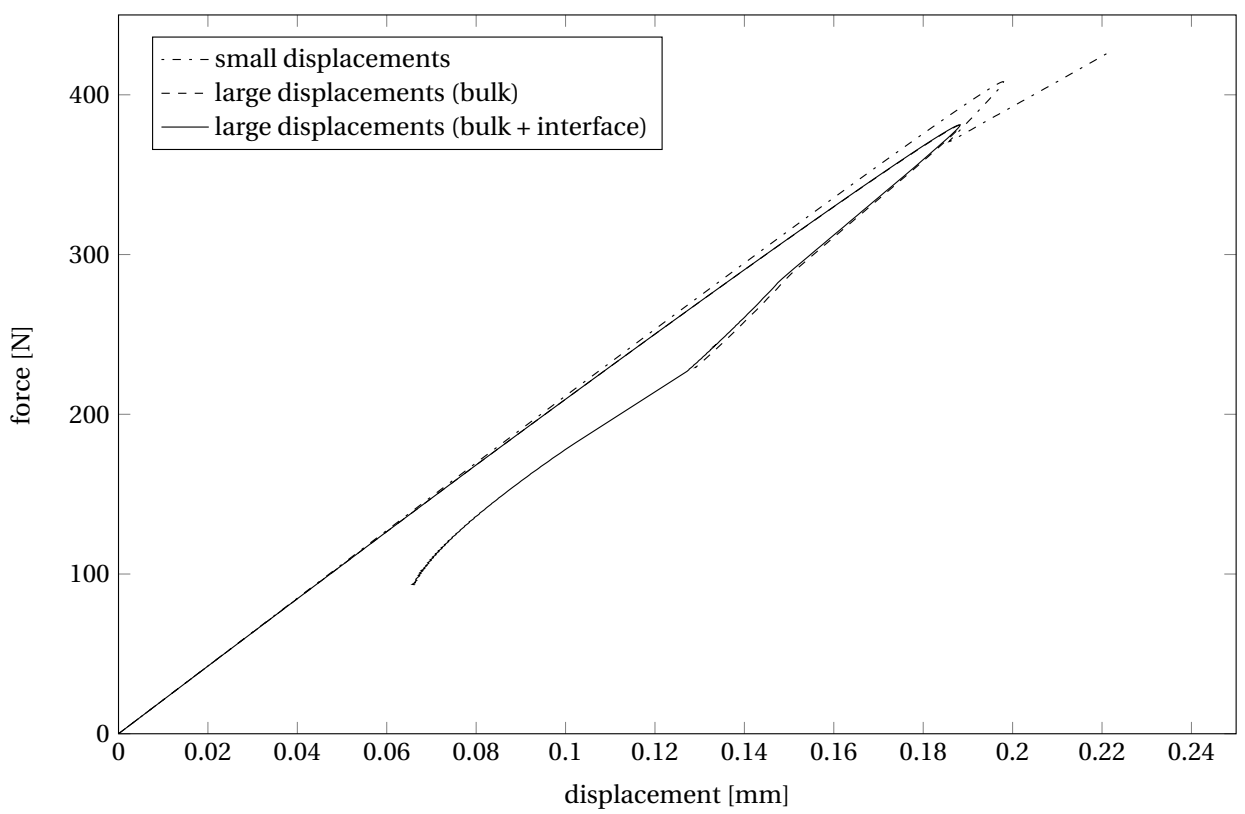

Figure 2.2: Load-displacement diagrams for large and small displacements. $L=15 \mathrm{~mm}, t_{\text {mid }}=2 t_{\text {outer }}=0.5 \mathrm{~mm}$. Material: E-glass $/ 913$.

\subsection{Material Properties}

Figures 2.4 and 2.5 show the load-displacement diagram for a two-sided clamped $[0 / \overline{90}]_{s}$ laminate E-glass $/ 913$ and IM7/8552 carbon/epoxy respectively. The material properties are listed in Table 2.1 and 2.2. The fine mesh is shown in Figure 2.3 and has a length of $15 \mathrm{~mm}$ and a height of $1 \mathrm{~mm}$. The middle ply is twice as thick as the outer plies. As will be shown in a later section, global buckling will not occur and the critical load at the peak of the diagram is not influenced by the boundary conditions.

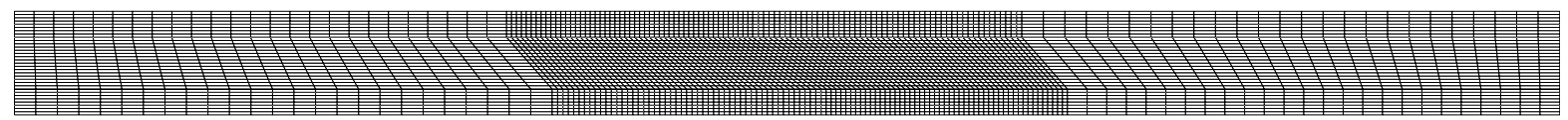

Figure 2.3: 2D Laminate mesh containing 5116 elements

The laminate acts linear-elastically until the horizontal interface elements start damaging (point A in Figure 2.4 and 2.5). When delamination continues, the failure load is reached (point B in Figure 2.4 and 2.5). When the inclined interface elements are totally damaged, it becomes harder to reach convergence in following iterations (point $\mathrm{C}$ in Figure 2.4 and 2.5). See figure 2.6 for the corresponding displacements. Note that the interface elements in the inclined crack are not yet completely damaged when the failure load is reached. The tractions and displacements corresponding to points B and C in Figure 2.4 are shown in Figure 2.7, for the material E-glass/913.

\begin{tabular}{|lcl|lcl|}
\hline \multicolumn{3}{|c|}{ Elasticity } & \multicolumn{3}{c|}{ Failure } \\
\hline$E_{1}$ & 43.9 & $\mathrm{GPa}$ & $F_{2 t}$ & 75.0 & $\mathrm{MPa}$ \\
$E_{2}$ & 15.4 & $\mathrm{GPa}$ & $F_{12}$ & 43.3 & $\mathrm{MPa}$ \\
$G_{12}$ & 4.34 & $\mathrm{GPa}$ & $G_{I c, m}$ & 0.25 & $\mathrm{~N} / \mathrm{mm}$ \\
$v_{12}$ & 0.3 & - & $G_{I I c, m}$ & 1.08 & $\mathrm{~N} / \mathrm{mm}$ \\
$v_{23}$ & 0.4 & - & $\eta$ & 1.0 & - \\
\hline
\end{tabular}

Table 2.1: Material properties for Hexcel E-glass/913 from [13] 


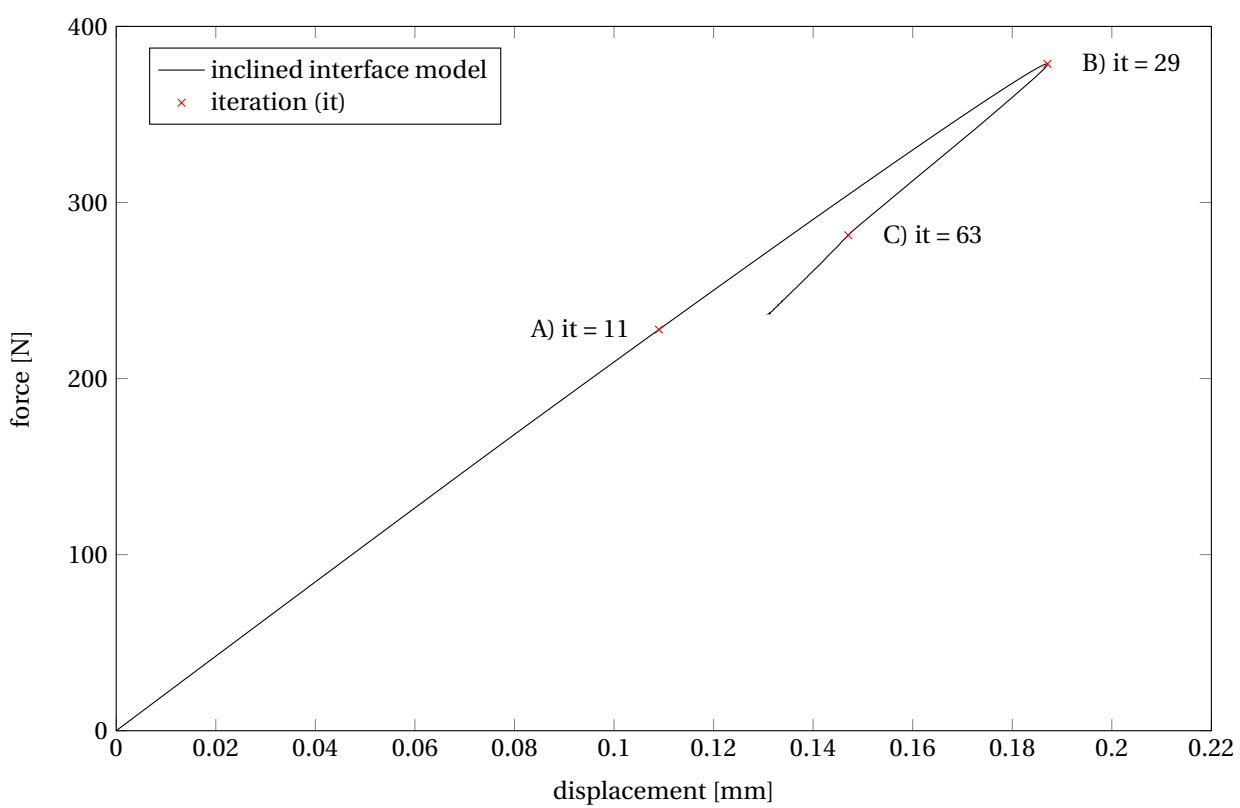

Figure 2.4: Load-displacement diagram. \pm 5000 elements, $L=15 \mathrm{~mm} t_{\text {mid }}=2 t_{\text {outer }}=0.5 \mathrm{~mm}$. Material: E-glass $/ 913$.

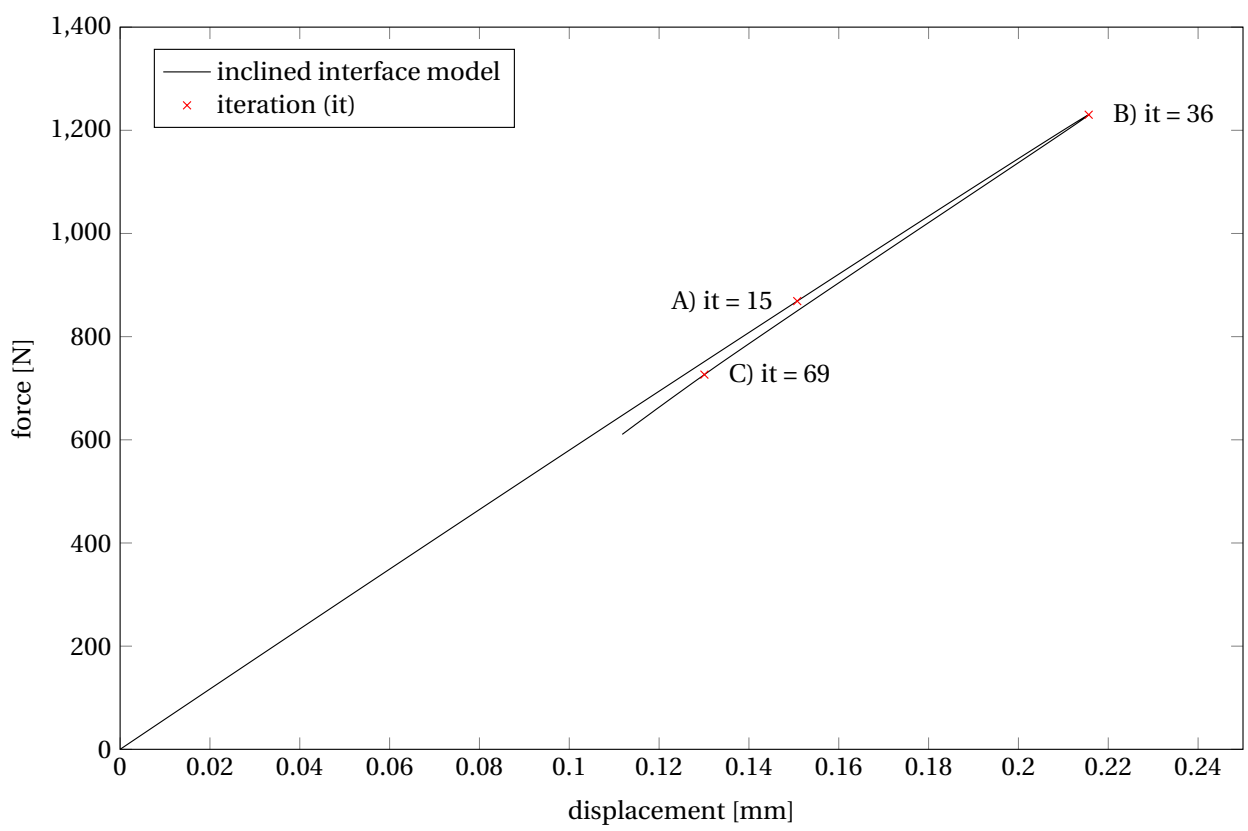

Figure 2.5: Load-displacement diagram. \pm 5000 elements, $L=15 \mathrm{~mm} t_{\text {mid }}=2 t_{\text {outer }}=0.5 \mathrm{~mm}$. Material: $\mathrm{IM} 7 / 8552$ carbon/epoxy.

\begin{tabular}{|lcl|lrl|}
\hline \multicolumn{3}{|c|}{ Elasticity } & \multicolumn{3}{c|}{ Failure } \\
\hline$E_{1}$ & 161.0 & $\mathrm{GPa}$ & $F_{2 t}$ & 60.0 & $\mathrm{MPa}$ \\
$E_{2}$ & 11.38 & $\mathrm{GPa}$ & $F_{12}$ & 45.0 & $\mathrm{MPa}$ \\
$G_{12}$ & 5.17 & $\mathrm{GPa}$ & $G_{I c, m}$ & 0.2 & $\mathrm{~N} / \mathrm{mm}$ \\
$v_{12}$ & 0.32 & - & $G_{I I c, m}$ & 1.0 & $\mathrm{~N} / \mathrm{mm}$ \\
$v_{23}$ & 0.4 & - & $\eta$ & 1.0 & - \\
\hline
\end{tabular}

Table 2.2: Material properties for IM7/8552 carbon/epoxy from [14] 

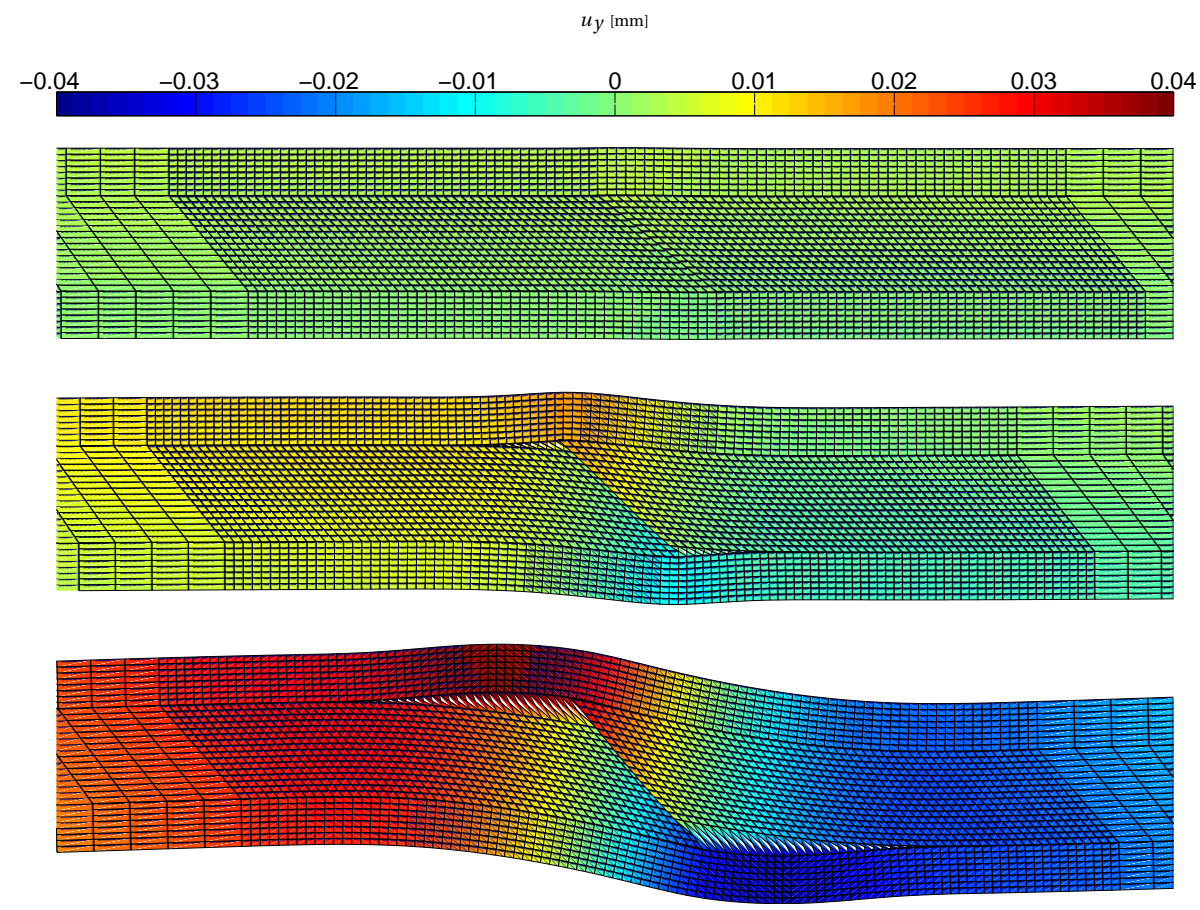

Figure 2.6: Displacements perpendicular to the load direction for the reference model corresponding to point A (above), B (middle) and $\mathrm{C}$ (below) in figure 2.4 (Scale factor $=5$ ). 
Tractions in Upper Interface Elements, Point B

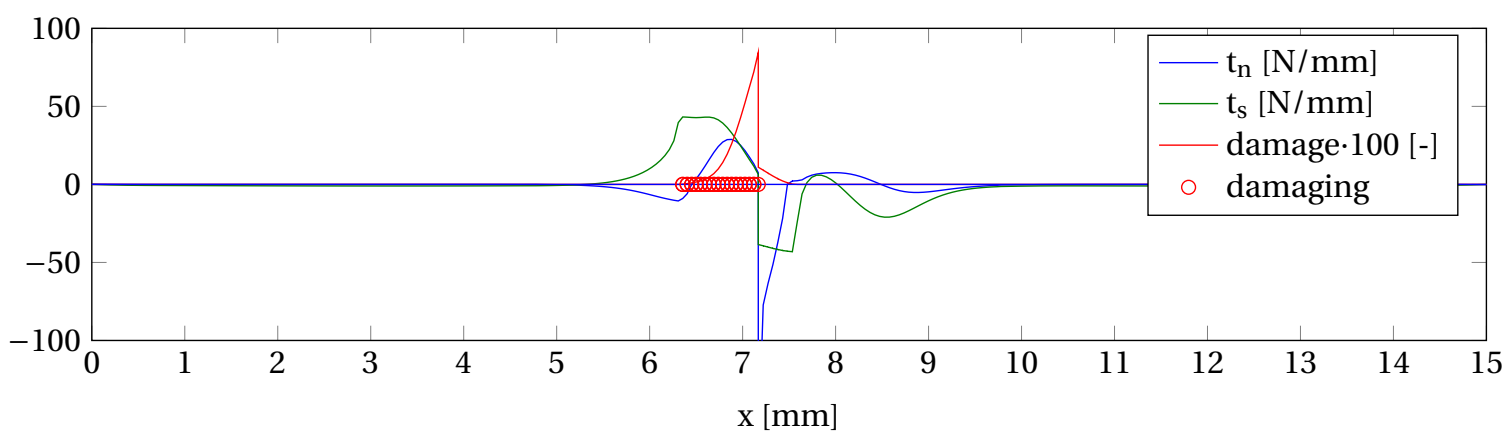

Jump in Upper Interface Elements, Point B

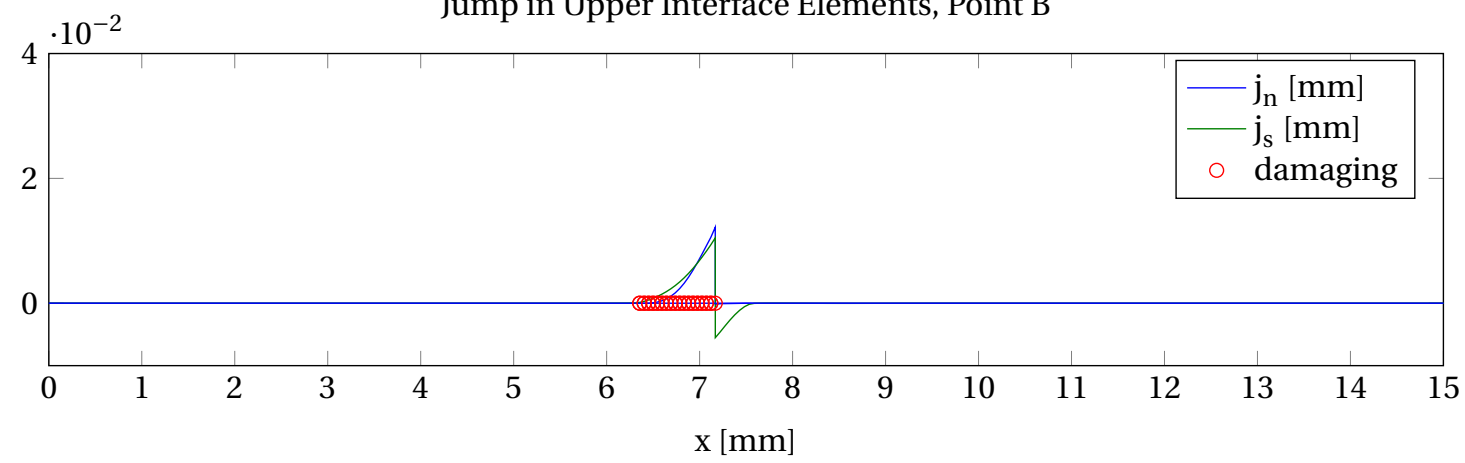

Tractions in Upper Interface Elements, Point C

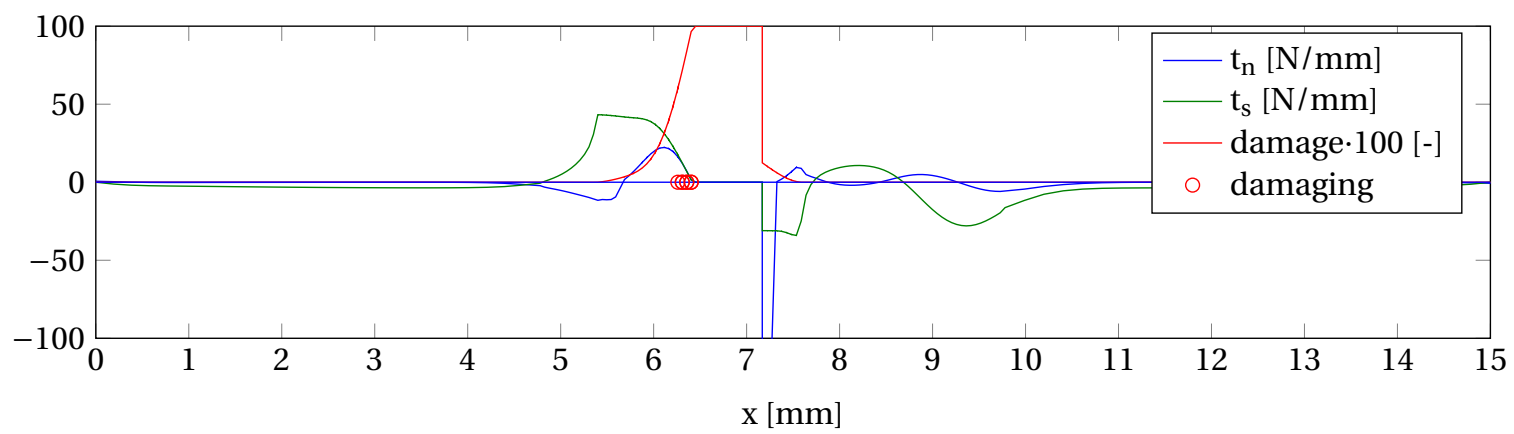

Jump in Upper Interface Elements, Point C

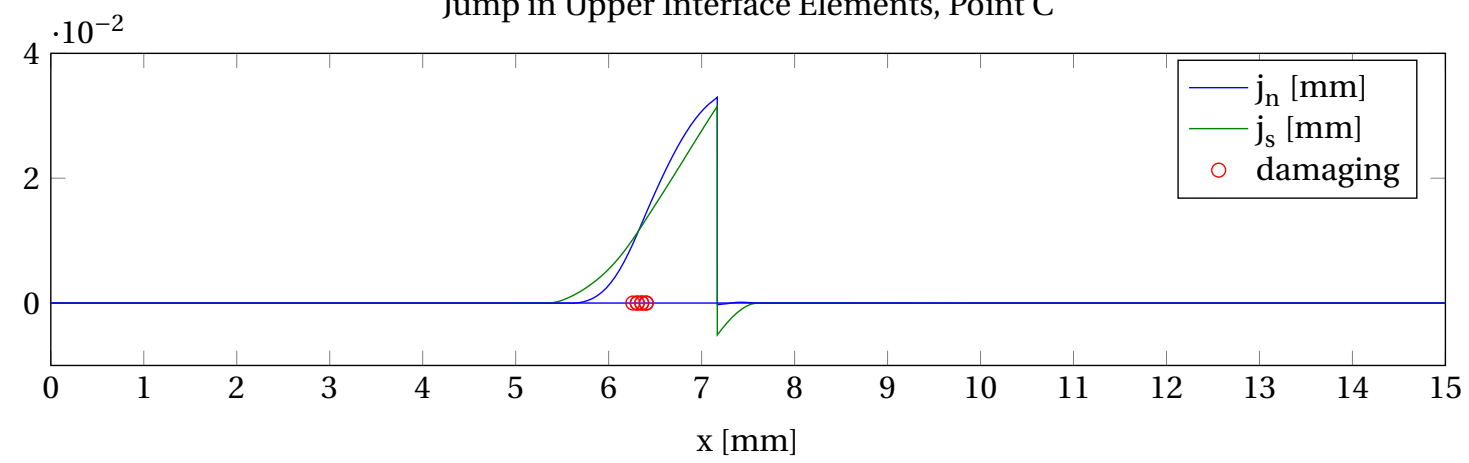

Figure 2.7: Tractions and jump in upper interface elements, corresponding to point C in Figure 2.4. $L=15 \mathrm{~mm} t_{\mathrm{mid}}=2 t_{\text {outer }}=0.5 \mathrm{~mm}$. Material: E-glass/913. 


\subsection{Buckling Length}

Since the reference model in 2D is a relatively slender structure, global buckling may occur if the buckling load is reached before delamination started. On the other hand, due to the symmetric mesh without imperfections, the model may fail in delamination while the failure load exceeds the buckling load. While the first situation does not provide the wanted failure load due to delamination, the second situation gives unreliable results. Therefore, the length of the laminate has to be limited to make sure that the buckling load is not exceeded when delamination occurs. In this section, an approximation for the buckling load of a two-sided clamped $[0 / \overline{90}]_{S}$ laminate E-glass/913 is investigated for varying dimensions to find these limitations.

For a high span-to-thickness ratio, the laminate can be considered slender. The failure load is found by the Euler buckling load for a clamped-clamped structure:

$$
F_{\text {Euler }}=\frac{\pi^{2} E I_{\text {eff }}}{l_{\text {buc }}^{2}}
$$

In which $l_{\text {buc }}=l / 2$ is the Euler buckling length for a two sided clamped structure and $E I_{\text {eff }}$ is the effective flexural stiffness for a symmetric laminate with unit width. When the assumption of a high span-to-thickness ratio is not valid, a finite shear rigidity has to be taken into account. Using a weighted average of the shear stiffness, $G$, and an approximation of the effective area $A_{\text {shear }}=\frac{5}{6} A$ as it is for rectangular isotropic crosssections, the Timoshenko failure load $F_{\text {Timoshenko becomes: }}$

$$
F_{\text {Timoshenko }}=G A_{\text {shear }}
$$

The Timoshenko failure load and the Euler failure load for a clamped beam are related by the standard solution [15]:

$$
\frac{1}{F_{\text {critical }}}=\frac{1}{F_{\text {Euler }}}+\frac{1}{F_{\text {Timoshenko }}}
$$

Numerical results for the buckling load are obtained by a mesh without interface elements where a small imperfection as shown in Figure 2.10 is added to the middle elements to trigger buckling. The load-displacement diagrams in Figure 2.8 are cut off after 50 iterations. The sudden change in slope of the diagram indicates global buckling. From the Figure and Table 2.3, it becomes clear that (2.5) leads to a better prediction of the buckling load then (2.3). Note that the maximum load of the numerical model that includes buckling is not necessarily a better approximation of the buckling load, since the applied force can still increase after global buckling occurred.

\begin{tabular}{l|lll}
\hline$L / h$ & 10 & 15 & 20 \\
\hline \hline$F_{\text {Euler }}[\mathrm{N}]$ & 1380 & 613.5 & 345.1 \\
$F_{\text {critical }}[\mathrm{N}]$ & 1033 & 533.6 & 318.3 \\
$F_{\text {numerical }}[\mathrm{N}]$ & 1034 & 533.7 & 318.8 \\
\hline
\end{tabular}

Table 2.3: Buckling load for varying length. $t_{\text {mid }}=2 t_{\text {outer }}=0.5 \mathrm{~m}$. Material: E-glass $/ 913$.

\begin{tabular}{l|lllllll}
\hline$t_{\text {mid }}[\mathrm{mm}]$ & 0.2 & 0.3 & 0.4 & 0.5 & 0.6 & 0.7 & 0.8 \\
$t_{\text {outer }}[\mathrm{mm}]$ & 0.4 & 0.35 & 0.3 & 0.25 & 0.2 & 0.15 & 0.1 \\
\hline \hline$F_{\text {critical }}[\mathrm{N}]$ & 562.3 & 558.9 & 550.0 & 533.6 & 507.7 & 469.7 & 416.9 \\
\hline
\end{tabular}

Table 2.4: Analytical buckling load for several ply-thickness ratios. $L=15 \mathrm{~mm}$. Material: E-glass/913.

Figure 2.9 and Table 2.4 present the analytical buckling load for several ply thickness ratios, $L / h=15$ and material properties from Table 2.1. The buckling mode is shown in Figure 2.10. Future simulations in 2D in this work will have similar dimensions. The obtained failure load will be compared to these results to make sure that global buckling does not occur before the failure load is reached. 


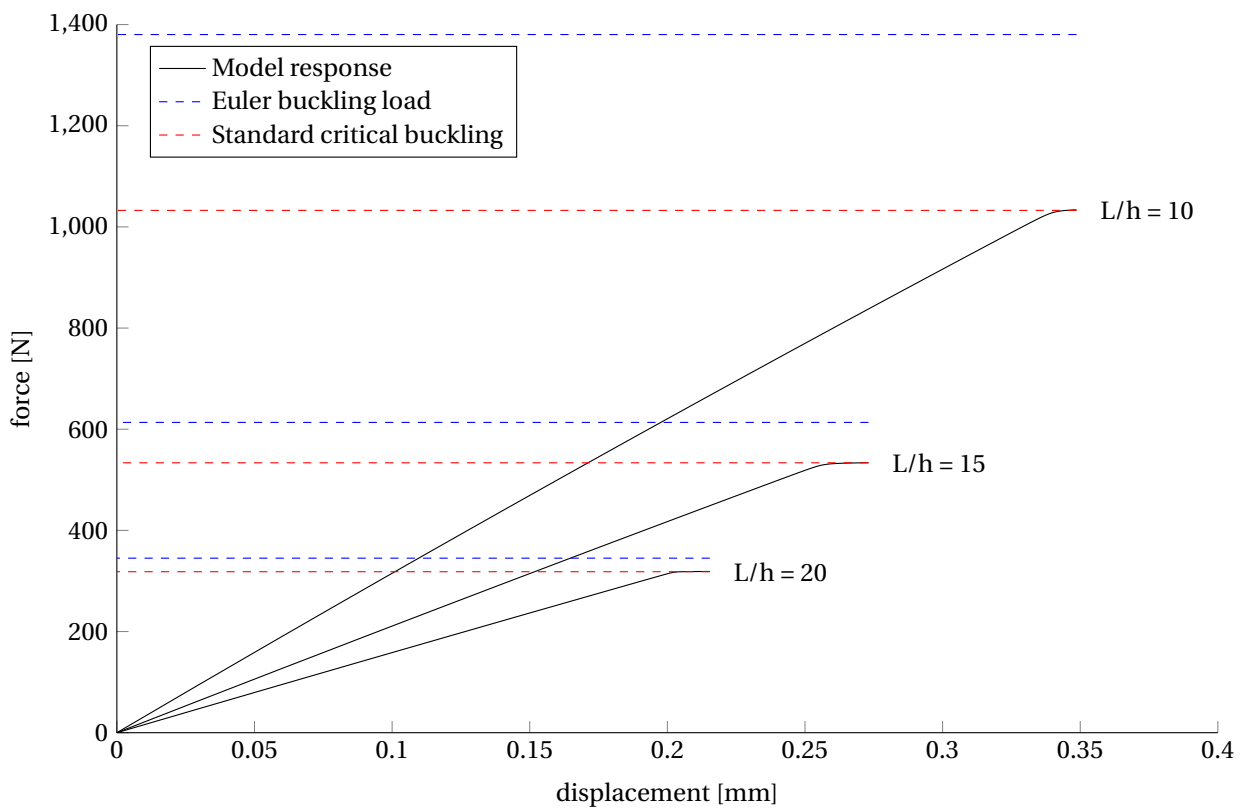

Figure 2.8: Load-displacement diagrams for varying length. $t_{\mathrm{mid}}=2 t_{\mathrm{outer}}=0.5 \mathrm{~mm}$. Material: E-glass $/ 913$.

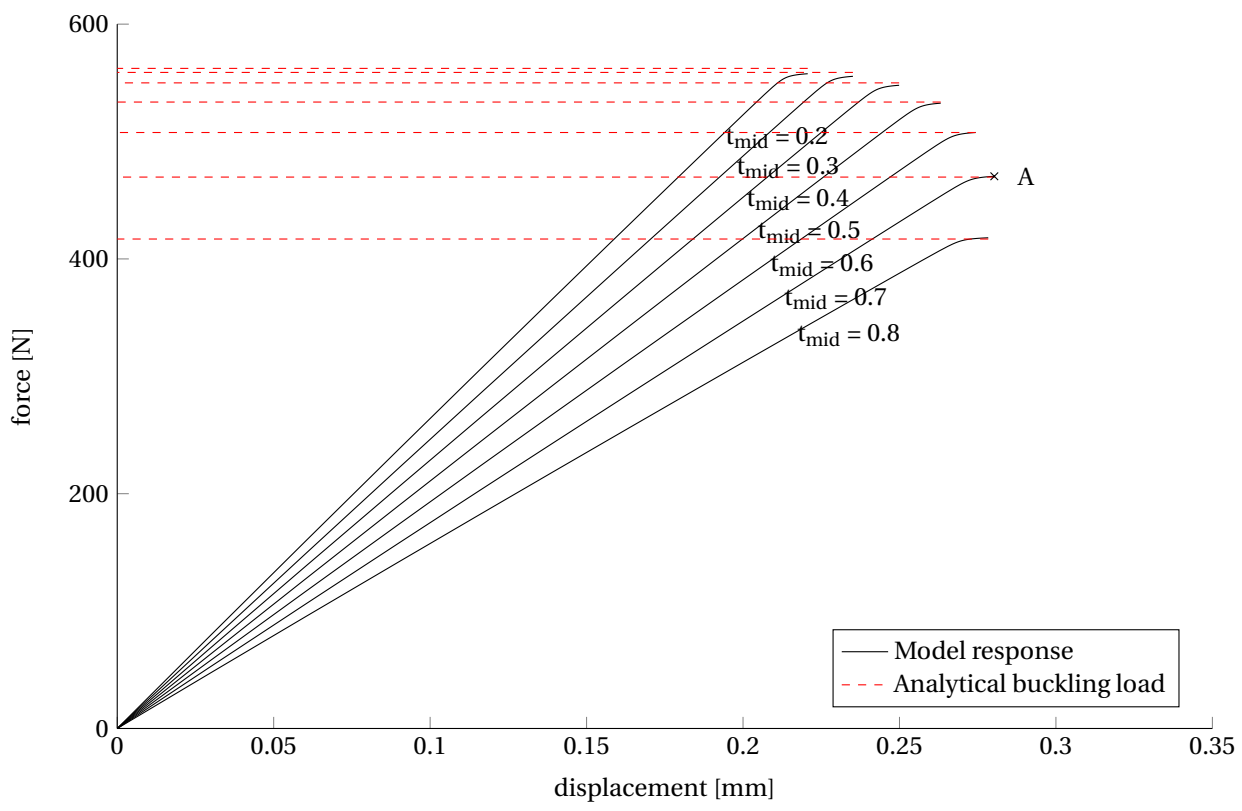

Figure 2.9: Global buckling for several ply-thickness ratios. $L=15 \mathrm{~mm}$. Material: E-glass $/ 913$.
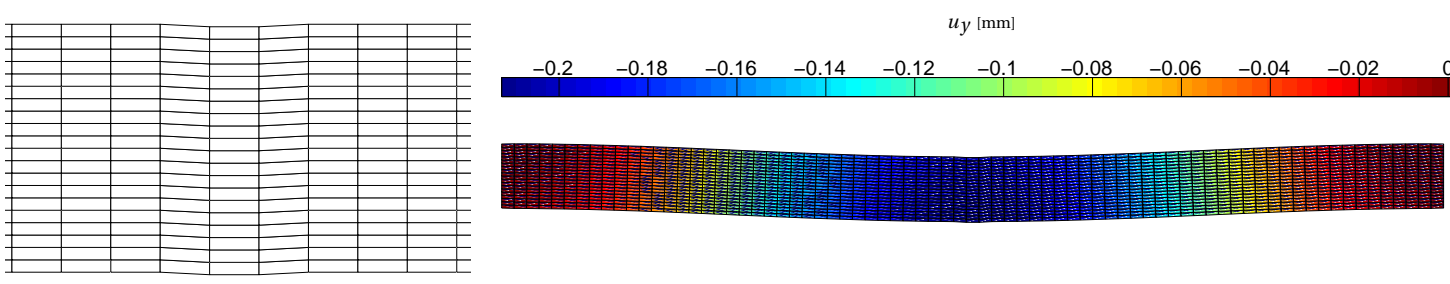

Figure 2.10: Mesh imperfection to trigger buckling (left) and buckling mode corresponding to point A in Figure 2.9 (right). 


\subsection{Boundary Conditions}

The boundary conditions to simulate an infinitely long laminate are shown in Figure 2.11. If the actual length of the model is too short, these assumptions may have an influence on the failure mechanism. Figure 2.12 shows an extreme situation where the crack opens due to the influence of reaction forces of the boundaries, working on the outer plies.

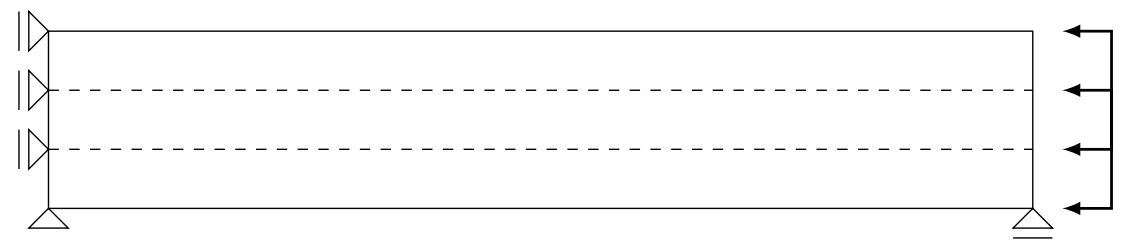

Figure 2.11: Boundary conditions to simulate an infinitely long laminate.

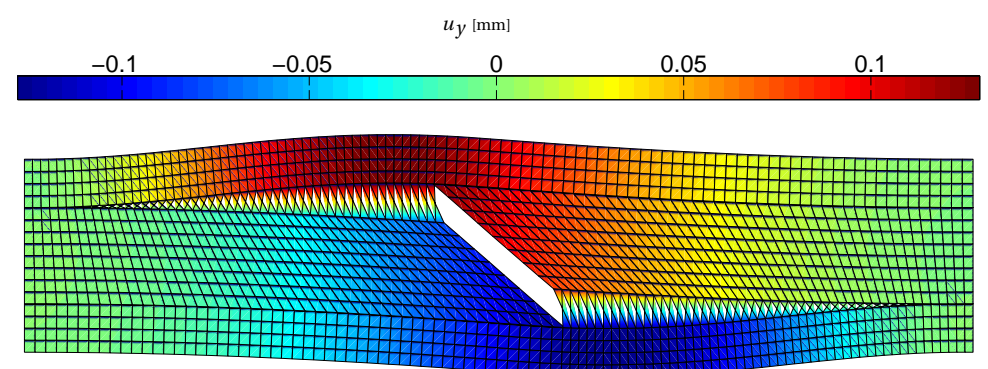

Figure 2.12: Failure mechanism influenced by boundary conditions. $L=5 \mathrm{~mm}, t_{\mathrm{mid}}=2 t_{\mathrm{outer}}=0.5 \mathrm{~mm}$. Material: E-glass $/ 913$.

Although no strict values are considered as limitations, visual inspection of the tractions in the horizontal interface elements in future simulations are carried out to make sure that the influence of the boundary conditions on the failure mechanism is negligible. 


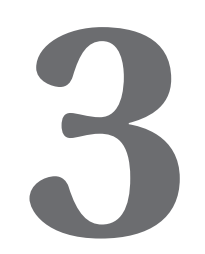

\section{TRANSFORMED INTERFACE MODEL}

To model the compressive laminate failure using a vertical crack plane, a transformation has to be applied in the cohesive law to account for the actual angle of the fracture plane. This so called 'transformed interface model' is shown in Figure 3.1 and is discussed in more detail in this section. Section 3.1 explains how this transformation is applied in 2D. In section 3.2, the transformed model is applied to a single ply under compressive loading. Since this is a relatively simple model and the failure mechanism is not influenced by interference with outer plies, the influence of the transformation on the behavior of the crack interface elements can be verified. Subsequently, the transformed model is applied to a two-sided clamped $[0 / \overline{90}]_{S}$ laminate in section 3.3. By looking into tractions and damage evolution in more detail, the model is compared to the reference model from Chapter 2.

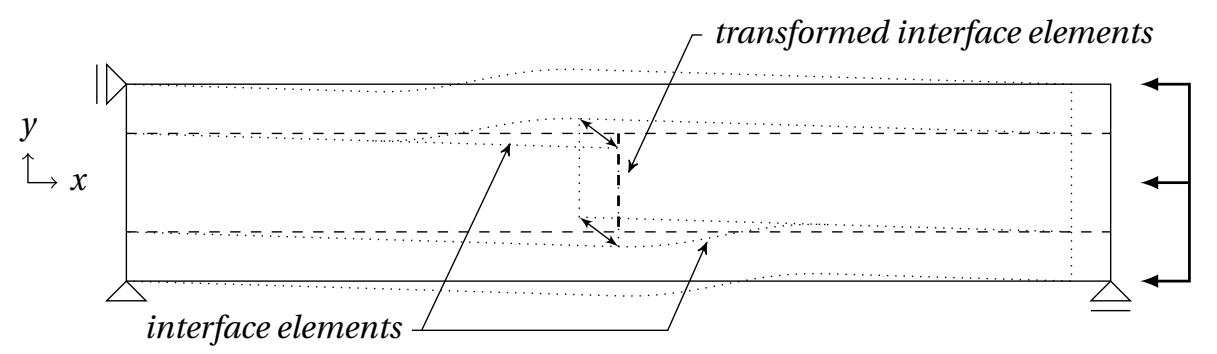

Figure 3.1: Schematic representation of modeling inclined compressive crack in a two-sided clamped $[0 / \overline{90}]_{S}$ laminate using transformed interface elements.

\subsection{Model Description}

The method of applying a transformation to the cohesive law of an interface element, referred to as the 'transformed interface model', is explained in this section for a 2D plane strain situation. An elementary ply as shown in Figure 3.2 is considered. The fiber orientation is perpendicular to the load direction, which means that the material is isotropic in the analysis plane. For pure compressive loading, the fracture plane $\alpha$ can be considered a material property $\alpha_{0}=53^{\circ}$.

Interface elements with the mixed mode damage law from Turon [3] serve as a basis for the transformed interface model. By using vertical interface elements for an inclined crack, the cohesive law (1.1) is applied in the inclined fracture plane rather than the local $\{n, s\}$-frame. Instead of adjusting the angle of the original rotation matrix, an extra rotation is added to the model. This way the transformation can be implemented as a wrapper around an existing cohesive law code. The transformation of the displacement jump $\overline{\mathbf{j}}$ from the local $\{n, s\}$-frame to $\overline{\mathbf{j}}^{*}$ in the inclined $\left\{n^{*}, s^{*}\right\}$-frame as shown in Figure 3.4 is done by the rotation matrix $\mathbf{R}_{\alpha}$ :

$$
\mathbf{R}_{\alpha} \overline{\mathbf{j}}=\left[\begin{array}{cc}
\cos (\alpha) & \sin (\alpha) \\
-\sin (\alpha) & \cos (\alpha)
\end{array}\right]\left[\begin{array}{c}
j_{n} \\
j_{s}
\end{array}\right]=\left[\begin{array}{c}
j_{n}^{*} \\
j_{s}^{*}
\end{array}\right]=\overline{\mathbf{j}}^{*}
$$




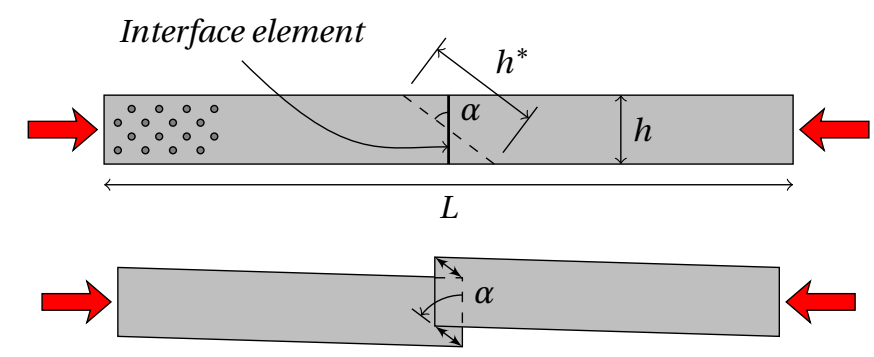

Figure 3.2: Model of compressive transverse cracking in an elementary ply.

In the $\left\{n^{*}, s^{*}\right\}$-frame, the cohesive law (1.1) is applied and the obtained traction vector $\overline{\mathbf{t}}^{*}$ and its linearization with respect to the displacement jump $\overline{\mathbf{T}}^{*}=\delta \overline{\mathbf{t}}^{*} / \delta \overline{\mathbf{j}}^{*}$ are transformed back into the local $\{n, s\}$-frame.

$$
\begin{gathered}
\mathbf{R}_{\alpha}^{\mathrm{T}} \overline{\mathbf{t}}^{*}=\left[\begin{array}{cc}
\cos (\alpha) & -\sin (\alpha) \\
\sin (\alpha) & \cos (\alpha)
\end{array}\right]\left[\begin{array}{c}
t_{n}^{*} \\
t_{s}^{*}
\end{array}\right]=\left[\begin{array}{c}
t_{n} \\
t_{s}
\end{array}\right]=\overline{\mathbf{t}} \\
\mathbf{R}_{\alpha}^{\mathrm{T}} \overline{\mathbf{T}}^{*} \mathbf{R}_{\alpha}=\left[\begin{array}{cc}
\cos (\alpha) & -\sin (\alpha) \\
\sin (\alpha) & \cos (\alpha)
\end{array}\right] \overline{\mathbf{T}}^{*}\left[\begin{array}{cc}
\cos (\alpha) & \sin (\alpha) \\
-\sin (\alpha) & \cos (\alpha)
\end{array}\right]=\overline{\mathbf{T}}
\end{gathered}
$$

$\overline{\mathbf{t}}$ and $\overline{\mathbf{T}}$ are then transformed in the global coordinates with (1.8) and (1.9). Since the element force vector and the element stiffness matrix in (1.10) and (1.11) are defined by an integral over the interface surface, the difference in length of the interface elements $h$ and $h^{*}$ in Figure 3.2 has to be taken into account with a length-scale factor.

$$
\begin{gathered}
\mathbf{f}_{\text {elem }}=\frac{h^{*}}{h} \int_{\Gamma_{i}} \mathbf{N}^{\mathrm{T}} \mathbf{t} d \Gamma=\frac{1}{\cos (\alpha)} \int_{\Gamma_{i}} \mathbf{N}^{\mathrm{T}} \mathbf{t} d \Gamma \\
\mathbf{K}_{\text {elem }}=\frac{h^{*}}{h} \int_{\Gamma_{i}} \mathbf{N}^{\mathrm{T}} \mathbf{T N} d \Gamma=\frac{1}{\cos (\alpha)} \int_{\Gamma_{i}} \mathbf{N}^{\mathrm{T}} \mathbf{T N} d \Gamma
\end{gathered}
$$

The position of the transformations in the algorithm for an interface element is shown in figure 3.3. Note that the transformation is independent of the cohesive law used. 
for every interface element

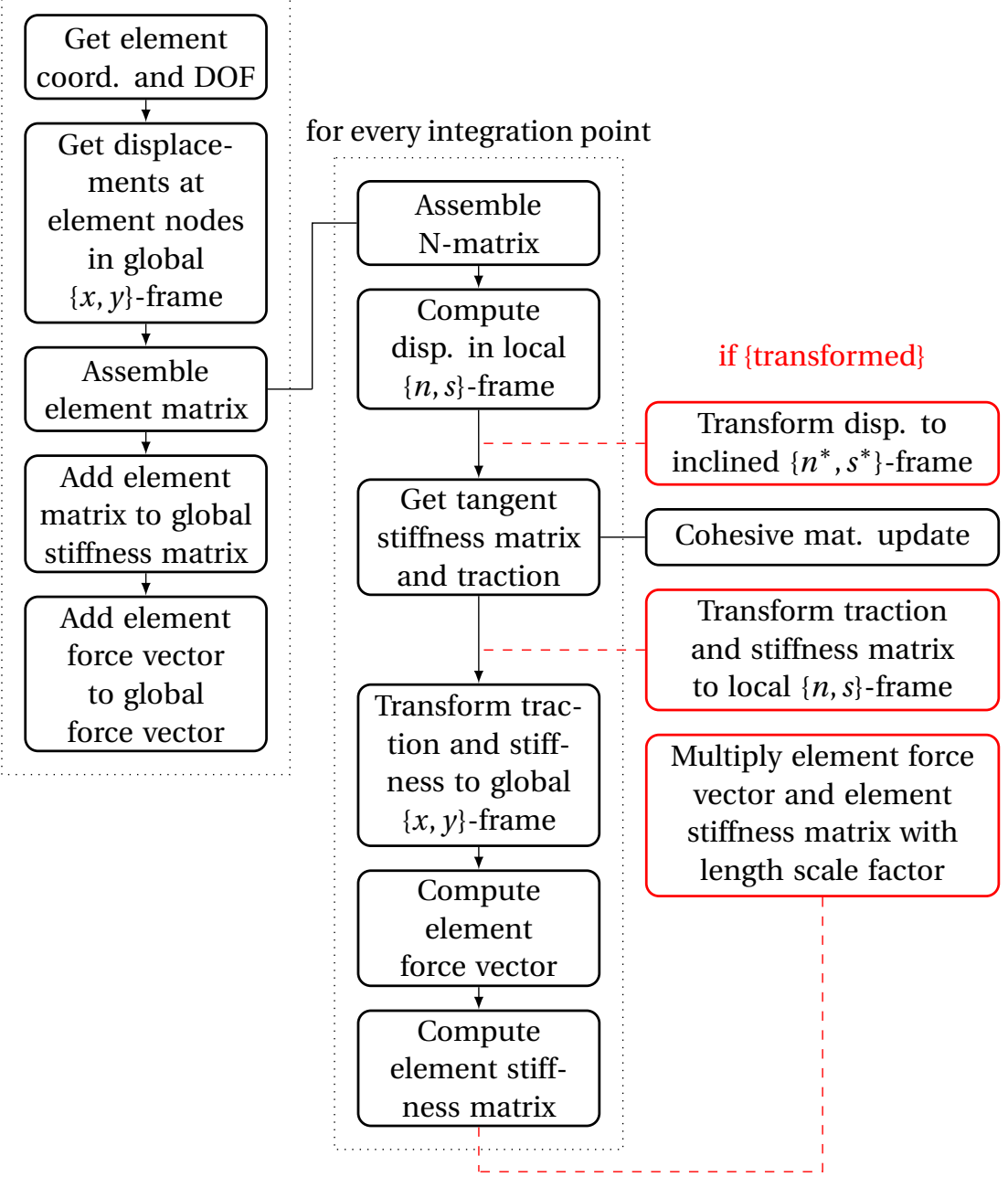

Figure 3.3: Schematic representation of interface model (black) and transformed interface model (red).

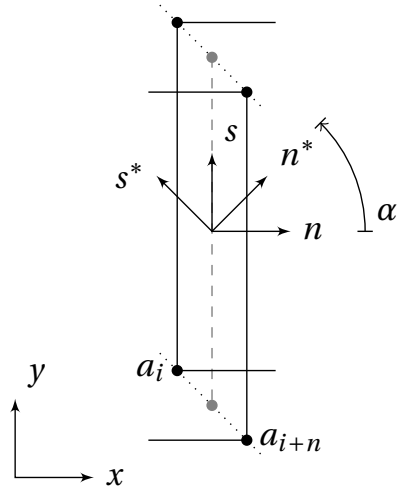

Figure 3.4: Interface element with local $\{n, s\}$-frame and inclined $\left\{n^{*}, s^{*}\right\}$-frame. 


\subsection{Single Ply}

For a transformed interface model of a single ply, the same material properties are used as described in section 2.2 albeit with an increased energy release rate to illustrate the cohesive behavior after crack initiation. To verify the model, a reference model as described in chapter 2 is used with a single ply. The inclined crack in the reference model is predefined by the topology of an interface element while the inclination is applied as a transformation in the cohesive law of a vertical interface element in the transformed model. As shown in Figure 3.5, 3.6 and Table 3.1, both models act the same, with a negligible difference of $0.0003 \mathrm{~N}$ in the failure load. The displacement of the center point of the laminate is shown in Figure 3.7.
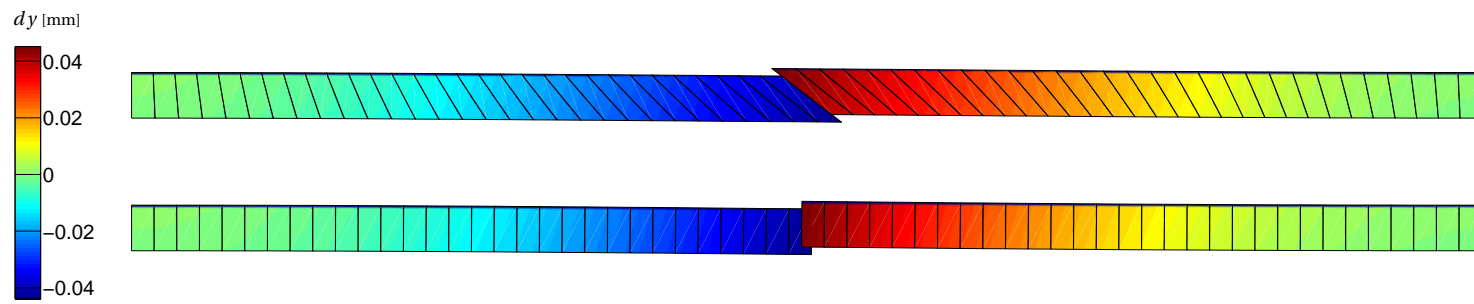

Figure 3.5: Vertical displacement in a single ply correpoinding to iteration 200 in Figure 3.6. Inclined interface model (above) and transformed interface model (below).

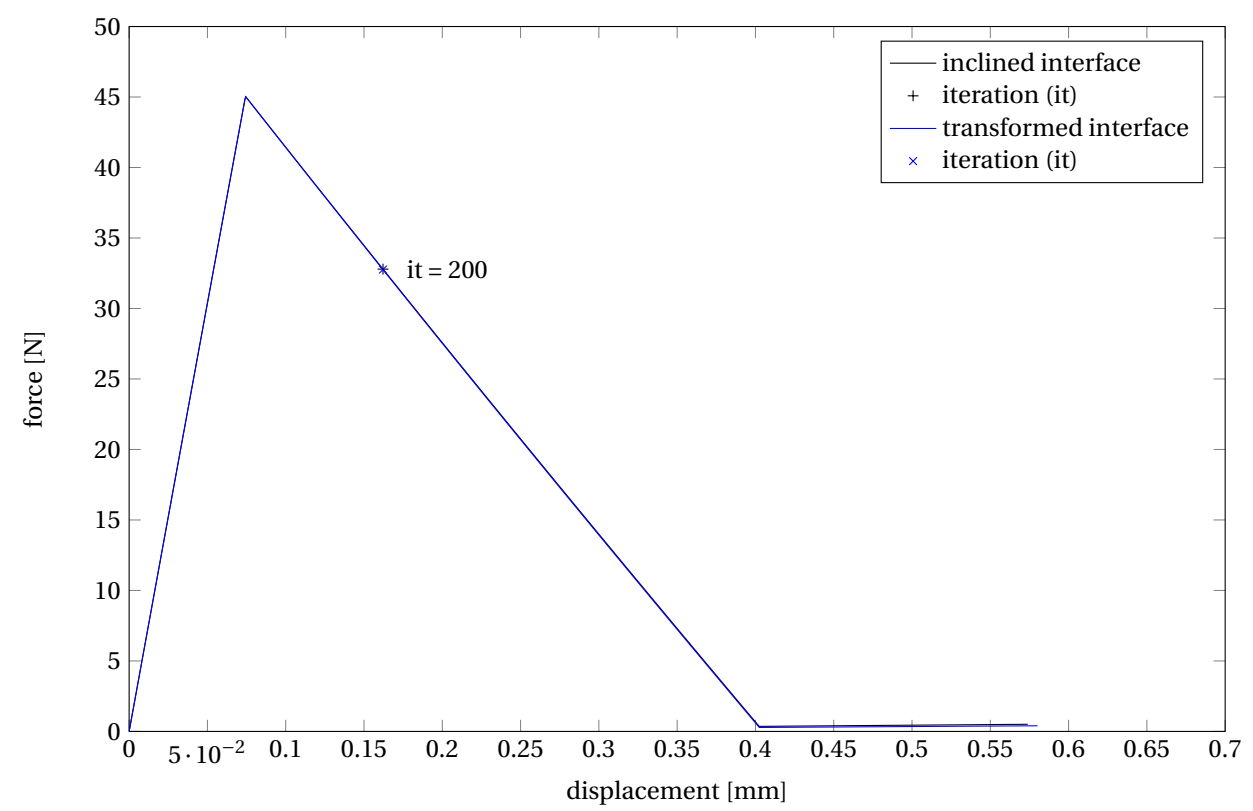

Figure 3.6: Load-displacement diagram for a single ply, inclined and transformed model. $L=15 \mathrm{~mm}, h=1 \mathrm{~mm}$. Material: E-glass 913 .

\begin{tabular}{ccc}
\hline & Inclined interface & Transformed interface \\
\hline \hline$F_{\text {failure }}[\mathrm{N}]$ & 45.0187 & 45.0188 \\
\hline
\end{tabular}

Table 3.1: Failure load for inclined and transformed interface model. $L=15 \mathrm{mn}$. Material properties from Table 3.2 . 


\begin{tabular}{|lll|lrl|}
\hline \multicolumn{3}{|c|}{ Elasticity } & \multicolumn{3}{c|}{ Failure } \\
\hline$E_{1}$ & 43.9 & $\mathrm{GPa}$ & $F_{2 t}$ & 75.0 & $\mathrm{MPa}$ \\
$E_{2}$ & 15.4 & $\mathrm{GPa}$ & $F_{12}$ & 43.3 & $\mathrm{MPa}$ \\
$G_{12}$ & 4.34 & $\mathrm{GPa}$ & $G_{I c, m}$ & 2.5 & $\mathrm{~N} / \mathrm{mm}$ \\
$v_{12}$ & 0.3 & - & $G_{I I c, m}$ & 10.8 & $\mathrm{~N} / \mathrm{mm}$ \\
$v_{23}$ & 0.4 & - & $\eta$ & 1.0 & - \\
\hline
\end{tabular}

Table 3.2: Material properties for Hexcel E-glass/913 from [13] with increased energy release rate.

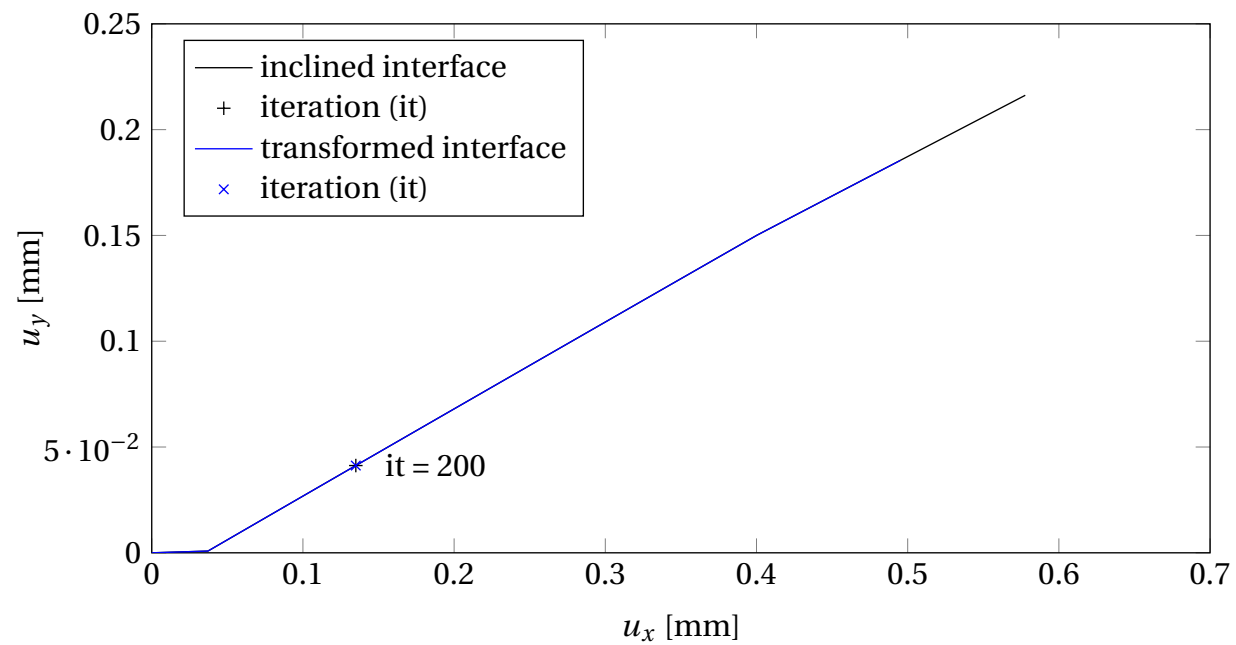

Figure 3.7: Displacement of center point of laminate in $x$ - and $y$-direction for inclined and transformed model. $L=15 \mathrm{~mm}, h=1 \mathrm{~mm}$. Material: E-glass 913.

\subsection{Laminate Analysis}

The effect of the transformation in the cohesive law of interface elements in a ply on modeling transverse compressive matrix cracking followed by delamination is assessed with the analysis of a $[0 / \overline{90}]_{s}$ laminate. Results obtained with the transformed cohesive law are compared to results from a model where the crack topology is predefined in the mesh. A mesh refinement study is done in section 3.3.1. In section 3.3.2, the behavior of the model is investigated by looking closer into the tractions and damage in the interface elements. The model is verified for varying ply thickness ratios in section 3.3.3.

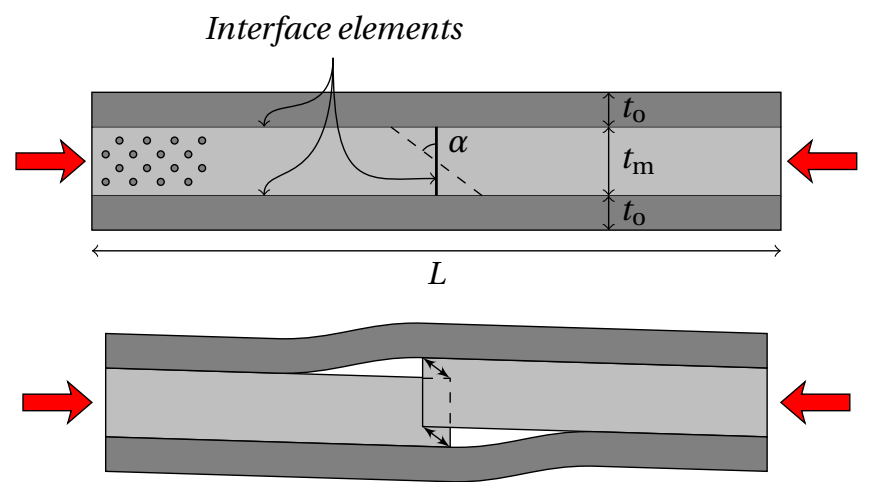

Figure 3.8: Model of compressive transverse cracking in a middle ply, followed by delamination.

A layup of three plies is considered as in Figure 3.8. The orientation of the fibers in the outer plies is in the load direction while the fibers in the middle ply are perpendicular to the load direction. Therefore, the material of the outer plies is anisotropic in the analysis plane with the material properties from Table 2.1. The vertical interface elements represent a matrix crack while the horizontal interface elements account for delamination. For pure compression, the fracture plane angle $\alpha$ of the matrix crack is set to a constant value 
$\alpha_{0}=53^{\circ}$. Using a damage based cohesive law for the interlaminar interface elements, snapback behavior is obtained for both the transformed interface model and the inclined interface model where the crack topology is predefined. Initially, both models act linearly. As the compressive stress in the plies increases, the failure criterion in the interfece elements in the middle ply is reached. These elements start to be damaged and the system becomes nonlinear. A wedge-shaped failure mechanism as obtained for a single ply in the previous section (Figure 3.5) is prevented by the outer plies. This results in an increasing tensile stress in the interlaminar interface elements. When the failure criterion in these elements is met, they start to be damaged and delamination can be observed. Even before the interface elements in the middle ply are fully damaged, total damage occurs in the interlaminar interface elements near the crack. The outer plies can no longer deal with the outward pushing force, so the failure load is reached. Post peak, delamination continues until the interface elements in the middle ply are also fully damaged. The displacements of both models are shown in Figure 3.9.
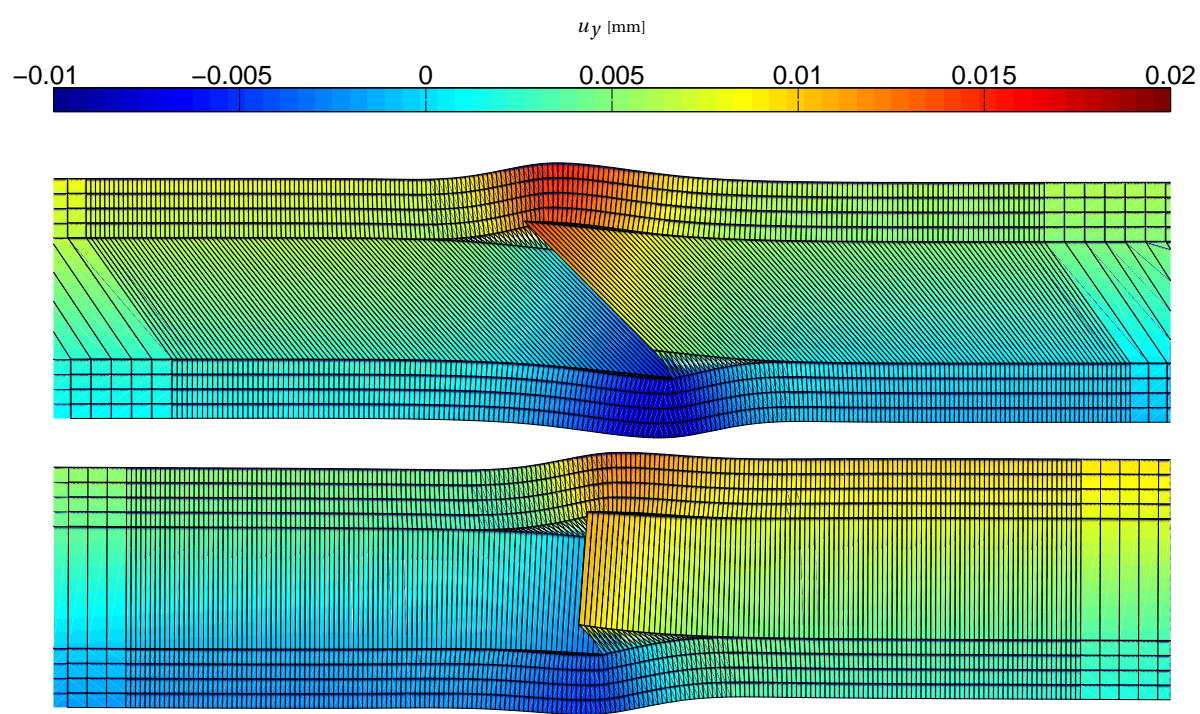

Figure 3.9: Vertical displacement in a $[0 / \overline{90}]_{s}$ laminate at failure. Inclined interface model (above) and transformed interface model (below) (scale factor $=10)$.

\subsubsection{Mesh Refinement}

So far, a single layer of elements per middle ply is used. Implicitly, the stresses and strains are assumed to be linearly distributed over the ply thickness. Since the stresses and strains might vary nonlinearly in the reference model, this could account for a difference in the failure load. In Figure 3.10 the load-displacement curves for one or more layers of elements per middle ply are compared. A dense mesh is still applied along the length of the ply to make sure that the cohesive model is a good approximation for delamination. The obtained failure loads are shown in Table 3.4. The failure load corresponding to the reference model with 20 layers of elements per middle ply is considered to be the most realistic value.

\begin{tabular}{l|lll}
\hline Elements through ply thickness & 1 & 5 & 20 \\
\hline \hline$F_{\text {failure, inclined }[\mathrm{N}]}$ & 411.5 & 383.7 & 378.7 \\
$F_{\text {failure, transformed }}[\mathrm{N}]$ & 419.9 & 416.0 & 416.1 \\
difference (\%) & 2.0 & 8.4 & 9.9 \\
\hline
\end{tabular}

Table 3.3: Failure loads for varying amount of elements through ply thickness. $L=15 \mathrm{~mm}, t_{\mathrm{mid}}=2 t_{\mathrm{outer}}=0.5 \mathrm{~mm}$. Material: E-glass $/ 913$.

The behavior of an inclined interface model with two or more layers of elements per ply, differs significantly from the behavior of a model with a single layer of elements per ply. This is not the case for the transformed interface model. By looking into the stress and strains of the interface elements and surrounding elements, this behavior is investigated in more detail in section 3.3.2. 

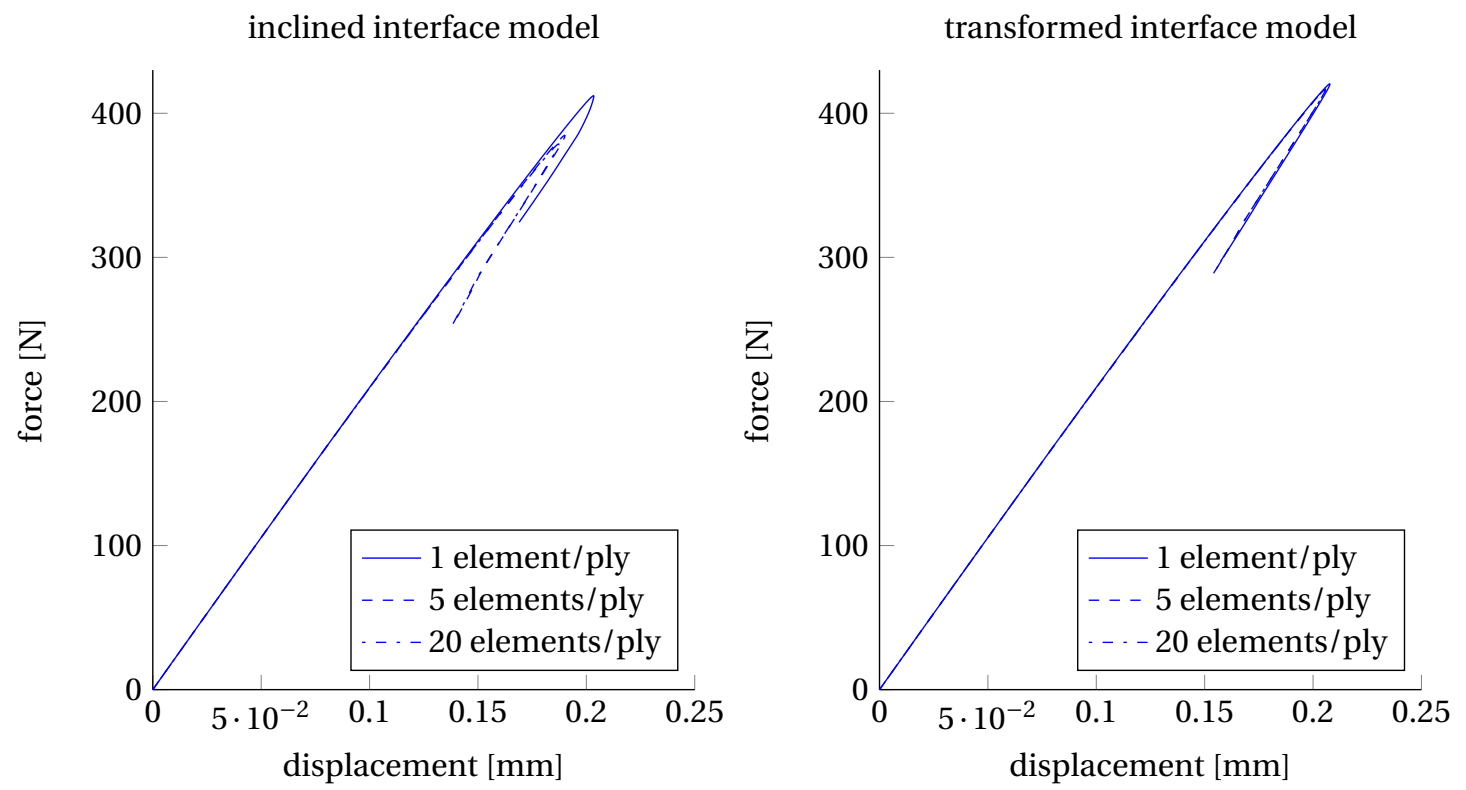

Figure 3.10: Load-displacement curves for varying amount of elements through ply thickness. $L=15 \mathrm{~mm}, t_{\text {mid }}=2 t_{\text {outer }}=0.5 \mathrm{~mm}$. Material: E-glass/913.

\subsubsection{Tractions in Interface Elements}

By plotting the stress $\sigma_{y y}$ using a single and multiple layers of elements per middle ply (Figure 3.12 and 3.14), it becomes clear that the stresses vary nonlinearly throughout the thickness of the ply. The resulting tractions in the horizontal interface elements are plotted in Figure 3.15 and 3.13 after the ninth iteration (point A in Figure 3.11). The blue arrows represent the size and direction of the traction. For simplicity, small displacement and rotations are assumed.

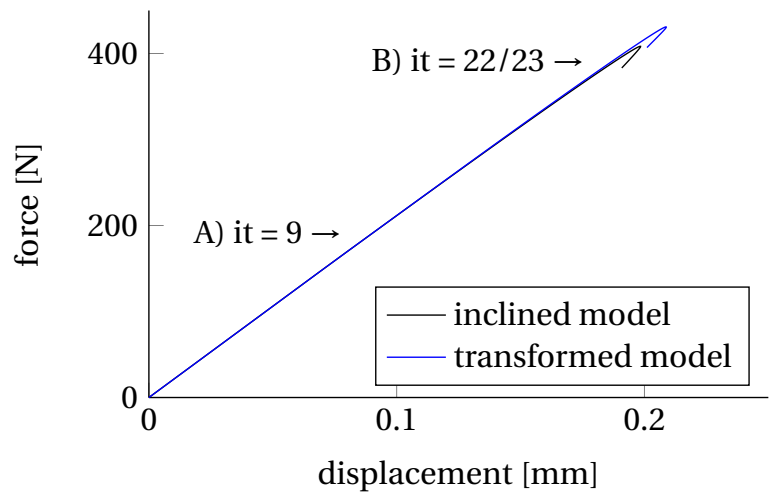

Figure 3.11: Load-displacement curves for both the incined and the transformed interface model using multiple layers of elements per ply. $L=15 \mathrm{~mm}, t_{\text {mid }}=2 t_{\text {outer }}=0.5 \mathrm{~mm}$. Material: E-glass $/ 913$.

The resulting force on the outer plies is initially the same for both models, while the distribution of the force is different. This results in more rapidly increasing damage in the interlaminar interface elements in the inclined interface model than in the transformed interface model as shown in Figure 3.16, leading to a lower failure load. For a complete laminate failure model not to be too expensive, a single layer of elements per ply is preferred. In that case, the distribution of the force on the outer plies in the transformed interface model equals the distribution of the force on the outer plies in the inclined interface model. Because of this, the failure load of a compressive loaded laminate with a single layer of elements per ply can be modeled well with a vertical crack plane and a transformation in the cohesive law. 


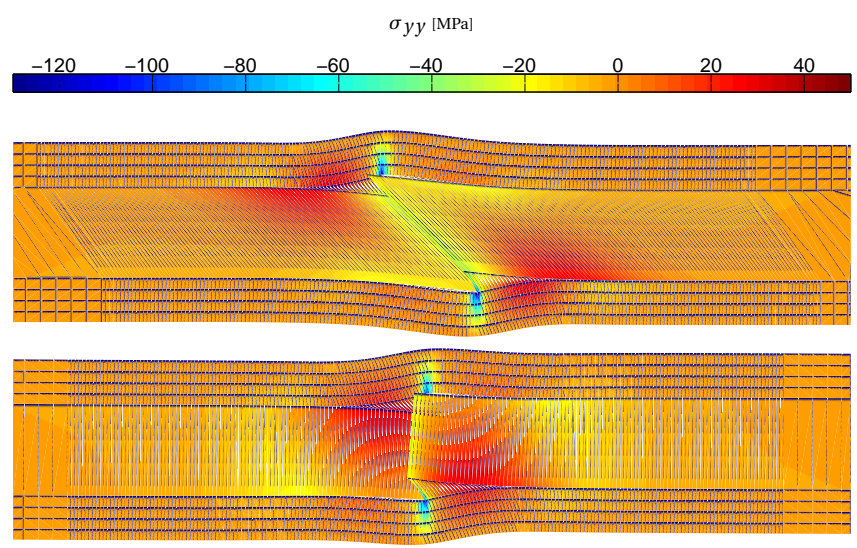

Figure 3.12: Stress $\sigma_{y y}$ in a [0/ $\left.\overline{90}\right]_{s}$ laminate at failure using a single layer of elements per middle ply. Inclined interface model (above) and transformed interface model (below) (Scale factor $=10$ ).
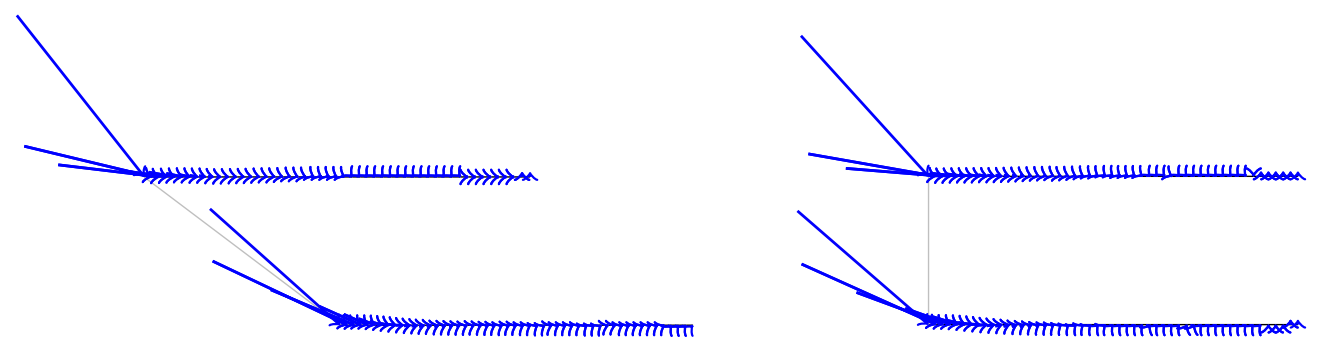

Figure 3.13: Tractions in interlaminar interface elements (left: inclined, right: transformed), using a single layer of elements per ply at the same load step.
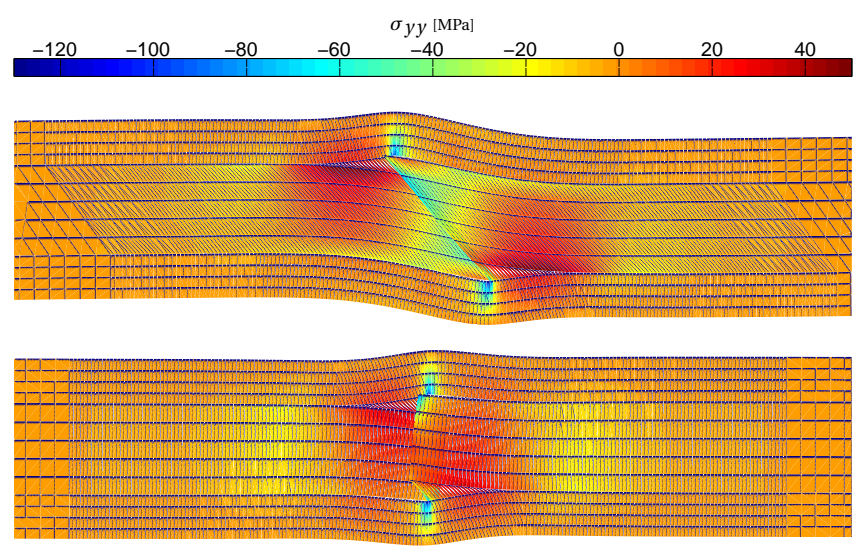

Figure 3.14: Stress $\sigma_{y y}$ in a $[0 / \overline{90}]_{s}$ laminate at failure using multiple layers of elements per middle ply. Inclined interface model (above) and transformed interface model (below) (Scale factor $=10$ ).
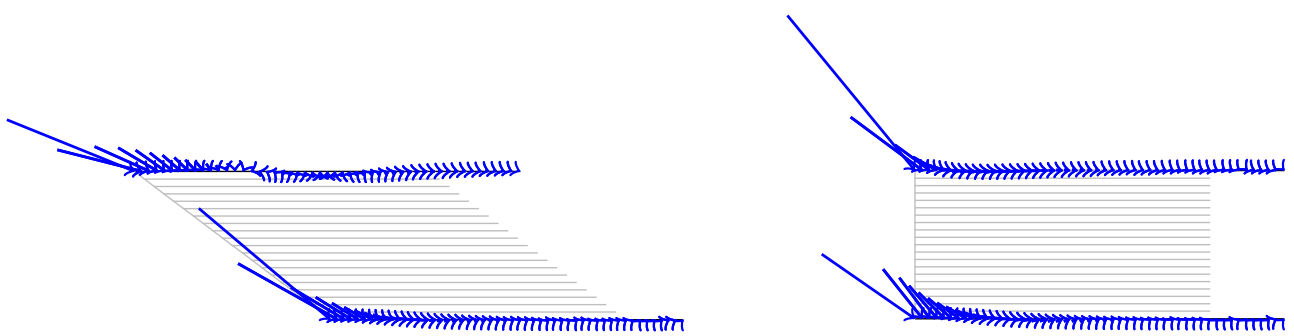

Figure 3.15: Tractions in interlaminar interface elements (left: inclined, right: transformed), using a multiple layers of elements per ply at the same load step (iteration 9 in Figure 3.11). 
Tractions and Damage, Point A

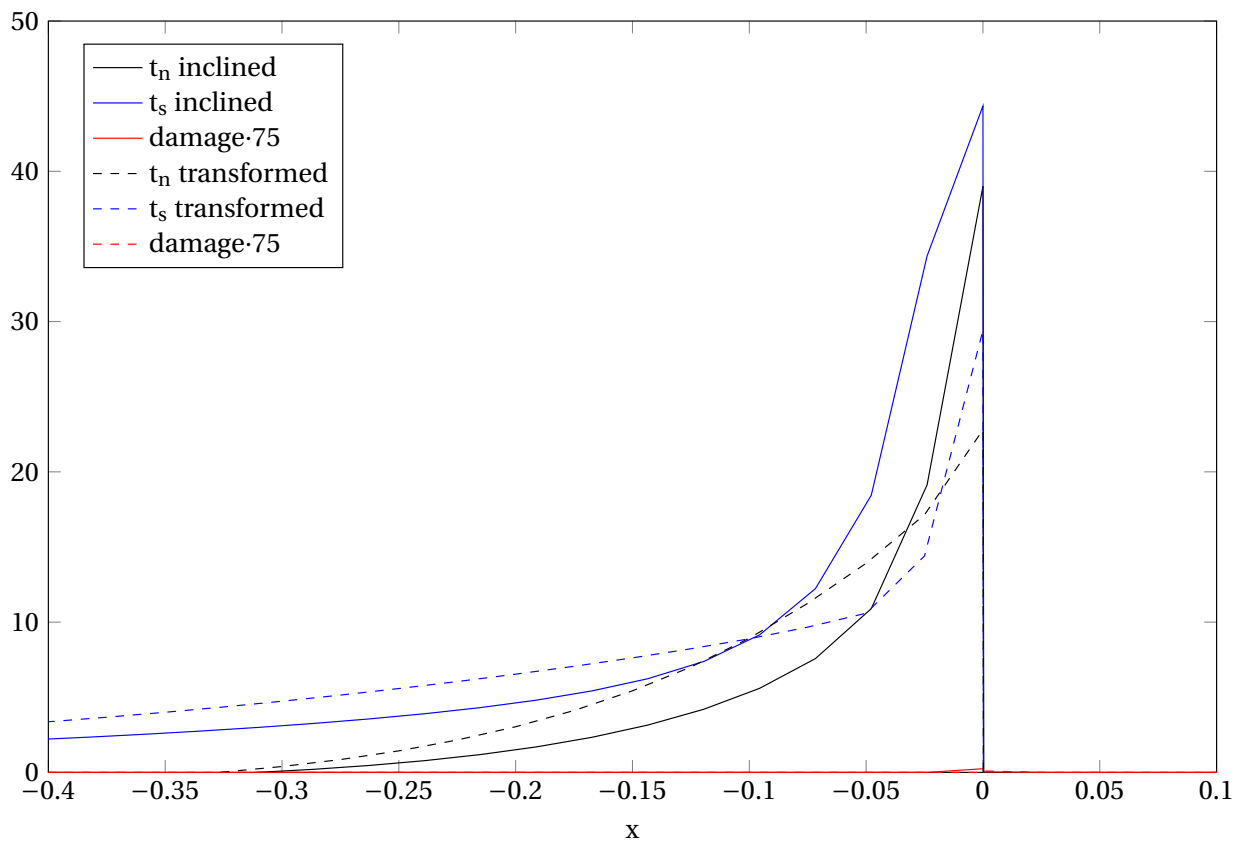

Tractions and Damage, Point B

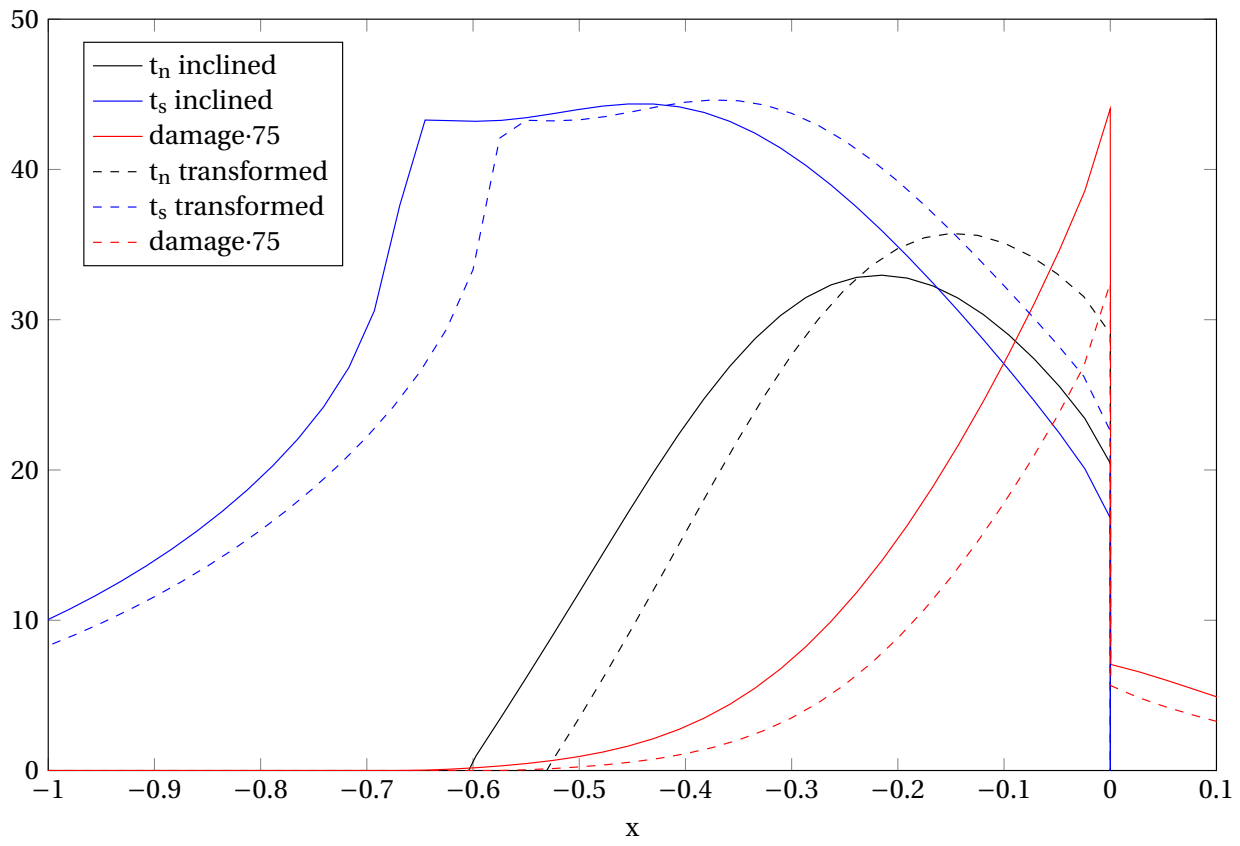

Figure 3.16: Traction and damage in interlaminar interface elements. $x=0$ refers to the intersection of the matrix crack with the interlaminar interface elements. 


\subsubsection{Ply Thickness Ratio}

Typical snapback behavior is shown in the load-displacement curves for several ply thickness ratios in Figure 3.17. The difference in failure load as described in the previous section can be observed.

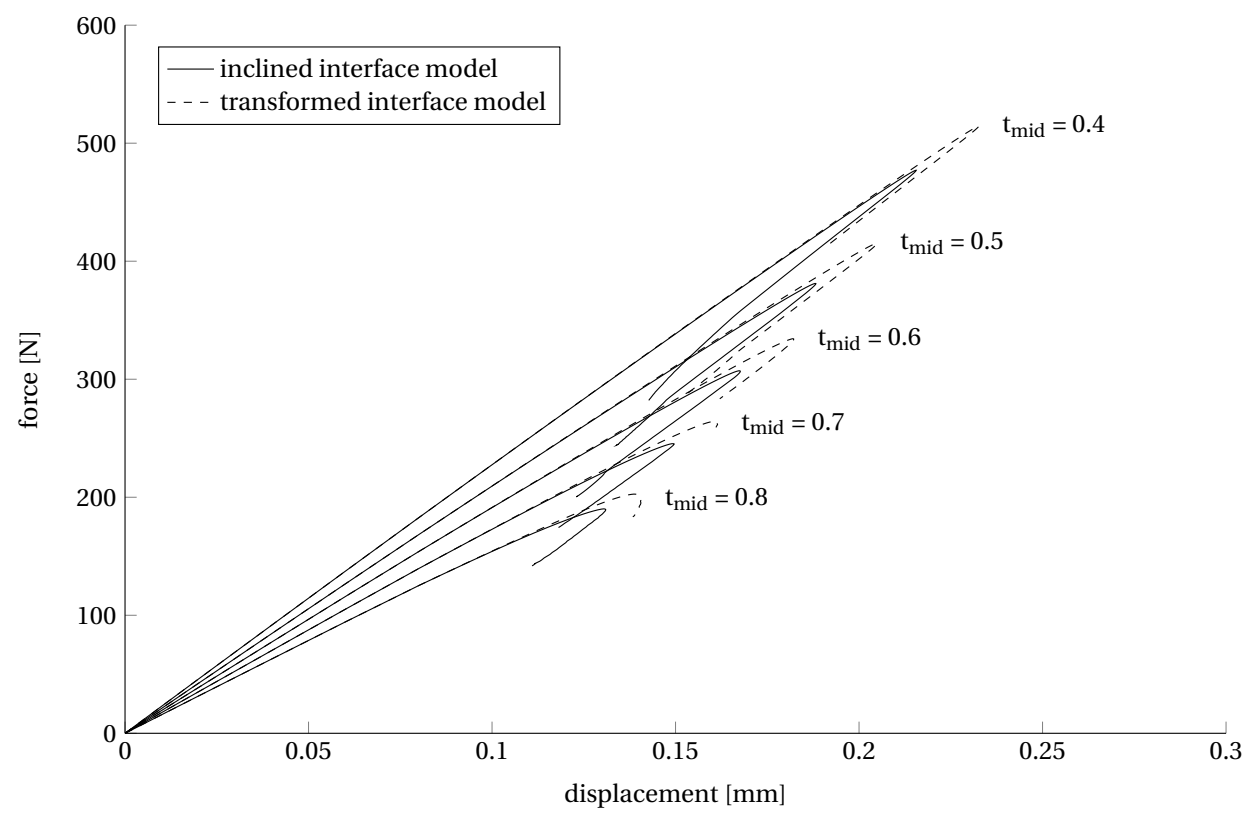

Figure 3.17: Load-displacement curves for varying ply thickness ratio using multiple layers of elements per ply. $L=15 \mathrm{~mm}, t_{\text {total }}=1$ mm. Material: E-glass/913.

\begin{tabular}{l|lllll}
\hline$t_{\text {mid }}[\mathrm{mm}]$ & 0.4 & 0.5 & 0.6 & 0.7 & 0.8 \\
$t_{\text {outer }[\mathrm{mm}]}$ & 0.3 & 0.25 & 0.2 & 0.15 & 0.1 \\
\hline \hline$F_{\text {failure, inclined }[\mathrm{N}]}$ & 476.4 & 381.2 & 307.5 & 245.6 & 190.2 \\
$F_{\text {failure, transformed }}[\mathrm{N}]$ & 514.9 & 416.0 & 335.3 & 265.1 & 203.1 \\
difference (\%) & 8.1 & 9.2 & 9.0 & 7.9 & 6.8 \\
\hline
\end{tabular}

Table 3.4: Failure loads for varying ply thickness ratio using multiple layers of elements per ply. $L=15 \mathrm{~mm}, t_{\text {total }}=1 \mathrm{~mm}$. Material: E-glass/913.

When a linear stress distribution is assumed, the transformed interface model is a good approximation of the inclined interface model. This can be seen in Figure 3.18 and Table 3.5 where the load displacement curves for several ply thickness are shown, using a single layer of elements in the middle ply. The difference in failure load is merely $2 \%$ and decreases when the influence of the outer plies on the failure load decreases.

\begin{tabular}{l|lllll}
\hline$t_{\text {mid }}[\mathrm{mm}]$ & 0.4 & 0.5 & 0.6 & 0.7 & 0.8 \\
$t_{\text {outer }}[\mathrm{mm}]$ & 0.3 & 0.25 & 0.2 & 0.15 & 0.1 \\
\hline \hline$F_{\text {failure, inclined }[\mathrm{N}]}$ & 511.8 & 411.7 & 332.2 & 264.3 & 203.9 \\
$F_{\text {failure, transformed }}[\mathrm{N}]$ & 522.1 & 419.9 & 336.6 & 265.8 & 204.1 \\
difference (\%) & 2.0 & 2.0 & 1.3 & 0.6 & 0.1 \\
\hline
\end{tabular}

Table 3.5: Failure loads for varying ply thickness ratio using a single layer of elements per ply. $L=15 \mathrm{~mm}, t_{\text {total }}=1 \mathrm{~mm}$. Material: E-glass/913. 


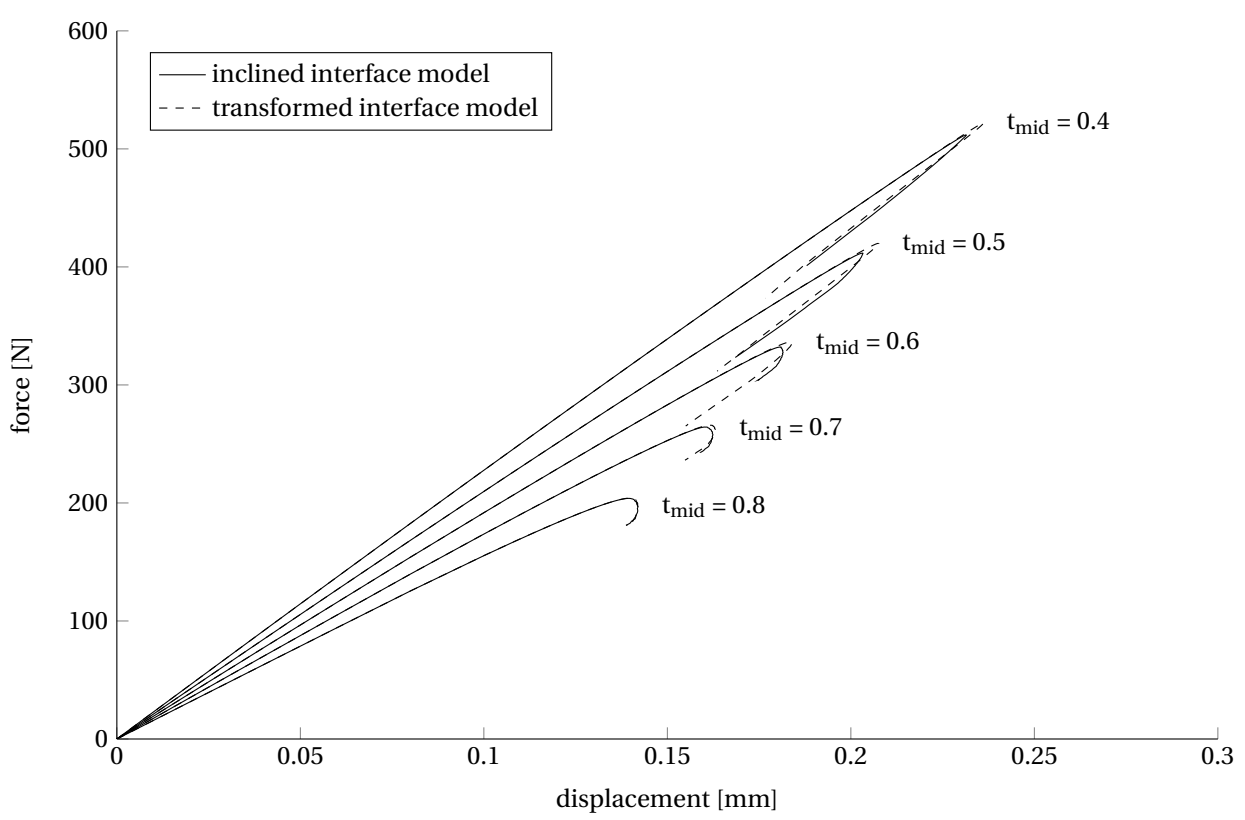

Figure 3.18: Load-displacement curves for varying ply thickness ratio using a single layer of elements per ply. $L=15 \mathrm{~mm}, t_{\text {total }}=1 \mathrm{~mm}$. Material: E-glass/913.

\subsubsection{Conclusion}

From the laminate analysis, it becomes clear that the transformation in the cohesive law is a good approximation of the compressive behavior of a laminate. The difference in the crack geometry when a vertical crack plane is used compared to a reference model where the inclined crack topology is predefined, result in a slightly higher failure load. This difference increases for an increasing influence of the outer plies on the failure load. Still, for relatively thick outer plies and a thin cracked middle ply, the difference in failure load is only a few percent.

Above holds when a single layer of elements per ply is used. Implicitly, a linear stress distribution through the thickness is thereby assumed. When the non-linear stress distribution is investigated in 2D, it is observed that the actual distribution of the stress in the plies in the transformed model is different compared to the reference model where the crack topology is predefined. A 3D model would become too expensive with multiple layers of elements per ply, especially when a crack runs through the elements. Therefore, in the following chapter the transformed method is restricted to failure models with a single layer of elements per ply. 



\section{4}

\section{D ANALYSiS}

A transformation in the cohesive law of an interface as tested in Chapter 3 will be implemented in a 3D model in this Chapter. The crack propagation is modeled with the phantom node method wherein the fiber orientation determines the direction of the crack propagation. Section 4.1 describes how the fracture plane angle is computed within the algorithm of the phantom node method and how thereafter a transformation to the constitutive law of the cohesive segment is applied. A mesh refinement study is done and the results are verified with a $[0 / \overline{90}]_{s} 2 \mathrm{D}$ model from Chapter 3. Furthermore, the influence of a varying fiber direction $\theta$ and fracture plane $\alpha$ are investigated in section 4.2 .2 and 4.2 .3 respectively.

\subsection{Model Description}

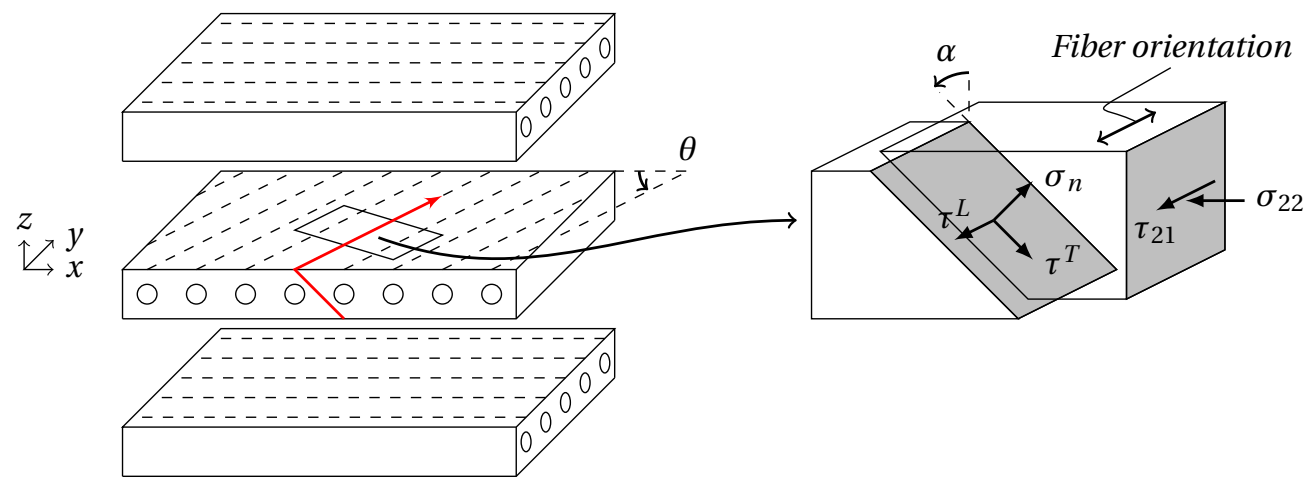

Figure 4.1: Fracture of a unidirectional lamina subjected to transverse compression and in-plane shear.

The laminate failure model by Van der Meer [2] serves as a basis of the so called 'transformed 3D model'. In Van der Meer's model, inter-fiber cracks are dealt with by the phantom node method as described in the introduction (section 1.4.3). Once a certain failure criterion is reached, a crack starts at a predefined location in the ply and propagates in the direction of the fiber orientation. This is taken into account by the angle $\theta$ that is input to the model. The fiber orientation $\theta$ and therewith the orientation of the fracture plane results in a combined compressive and shear stress in the cracked interface as shown in Figure 4.1. The fracture plane angle $\alpha$ for a combined compressive and shear stress is dependent on the actual $\sigma_{22} / \tau_{21}$-ratio. Therefore, the fracture plane angle $\alpha$ has to be found and initialized for every element that is crossed by the crack path before the cohesive law is applied in the phantom node method. This is schematically represented in the global algorithm in Figure 4.2.

The failure criterion LaRC03\#1 by Davila [16] is used for the initiation of a new crack segment as well as for determining the fracture plane angle. The failure index FI is related to the longitudinal and transverse shear strength $S^{L}$ and $S^{T}$ and the effective shear stresses $\tau_{\text {eff }}^{L}$ and $\tau_{\text {eff }}^{T}$ 


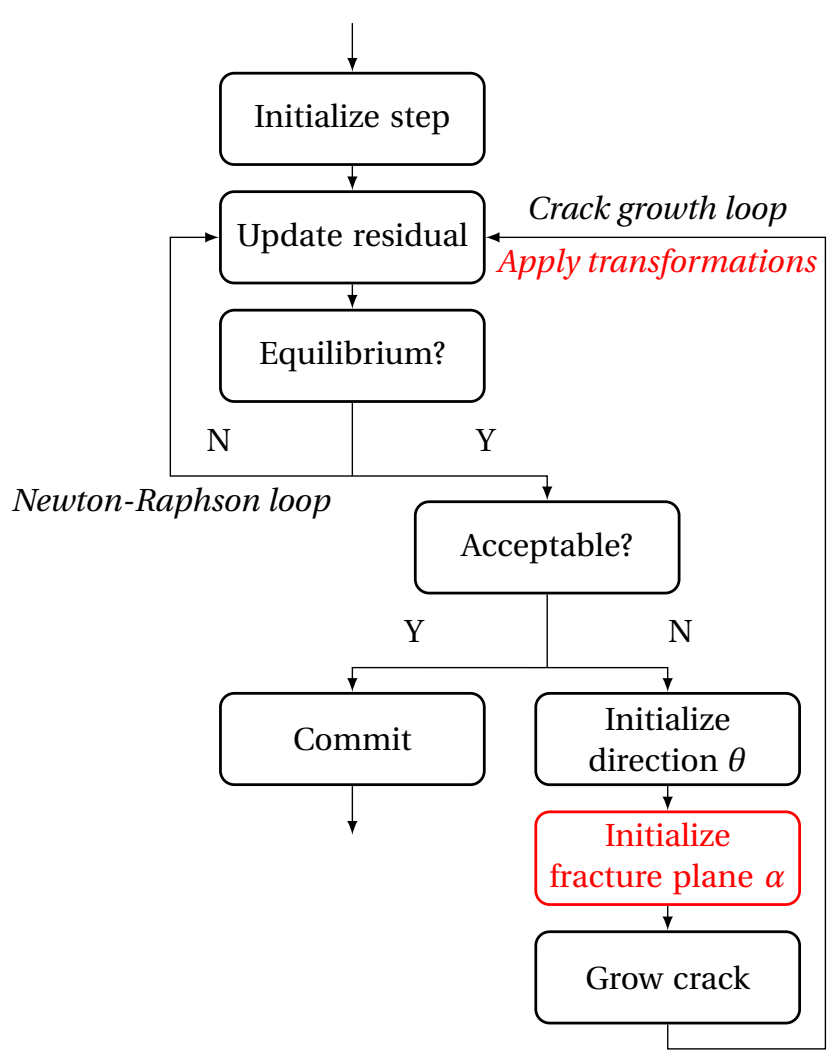

Figure 4.2: Position initialization $\alpha$ and transformations (red) and crack growth model in global algorithm.

$$
\mathrm{FI}_{\mathrm{M}}=\left(\frac{\tau_{\text {eff }}^{T}}{S^{T}}\right)^{2}+\left(\frac{\tau_{\text {eff }}^{T}}{S_{\text {is }}^{L}}\right)^{2} \leq 1
$$

The subscript $M$ indicates matrix failure. With $\alpha, \tau^{L}, \tau^{T}, \sigma_{n}, \tau_{21}$ and $\sigma_{22}$ as shown in Figure 4.1, the effective shear strengths $\tau_{\text {eff }}^{L}$ and $\tau_{\text {eff }}^{T}$ are defined as:

$$
\begin{gathered}
\tau_{\text {eff }}^{T}=\left\langle\left|\tau^{T}\right|+\eta^{T} \sigma_{n}\right\rangle=\left\langle-\sigma_{22} \cos \alpha\left(\sin \alpha-\eta^{T} \cos \alpha\right)\right\rangle \\
\tau_{\text {eff }}^{L}=\left\langle\left|\tau^{L}\right|+\eta^{L} \sigma_{n}\right\rangle=\left\langle\cos \alpha\left(\left|\tau_{21}\right|+\eta^{L} \cos _{22} \cos \alpha\right)\right\rangle
\end{gathered}
$$

where the terms $\eta^{T}$ and $\eta^{L}$ are referred to as coefficients of transverse and longitudinal influence, respectively, and the operand $\langle x\rangle=\frac{1}{2}(x+|x|)$. It is assumed that the longitudinal shear strength equals the transverse shear strength to be consistent with the cohesive model, which has only one shear strength parameter.

$$
S^{T}=S^{L}
$$

The compressive strength $Y^{C}$ and the coefficients of transverse and longitudinal influence can then be found using the fracture plane angle $\alpha_{0}$ for pure compression:

$$
\begin{gathered}
Y^{C}=\frac{S}{\cos \alpha_{0}\left(\sin \alpha_{0}+\frac{\cos \alpha_{0}}{\tan 2 \alpha_{0}}\right)} \\
\eta^{T}=\frac{-1}{\tan 2 \alpha_{0}}
\end{gathered}
$$




$$
\eta^{L}=-\frac{S^{L} \cos 2 \alpha_{0}}{Y^{C} \cos ^{2} \alpha_{0}}
$$

According to Puck [6], the fracture plane for pure compression $\alpha_{0}$ is assumed to be 53 degrees according to Puck [6]. For other $\sigma_{22} / \tau_{21}$-ratios, the correct angle of the fracture plane is the one that maximizes the failure index (FI). As done by Davila [16], this angle is obtained by searching for the maximum of the FI within a loop over the range of possible fracture angles: $0<\alpha<\alpha_{0}$. A graphical representation of matrix failure envelopes at various fracture angles is shown in Figure 4.3.

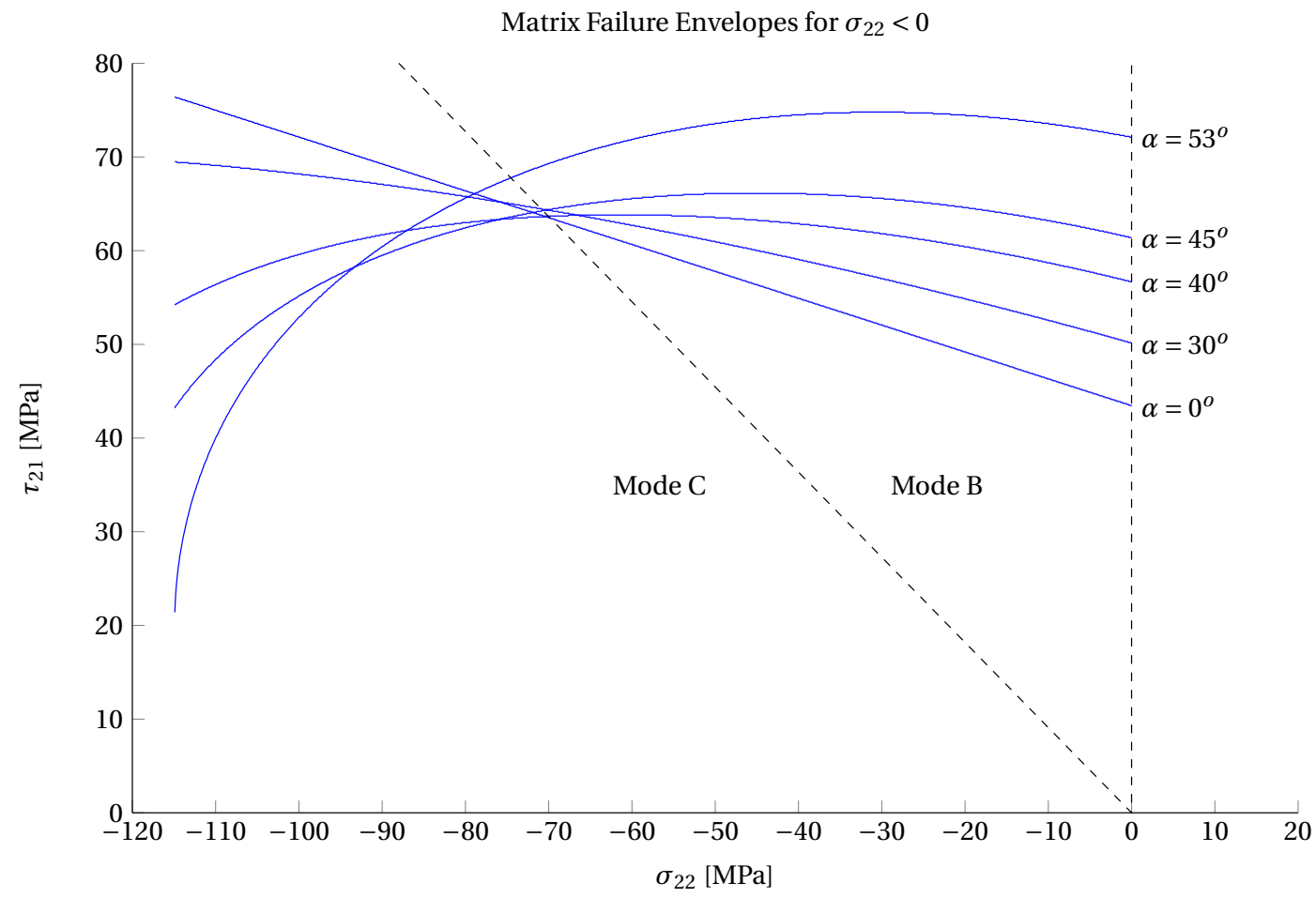

Figure 4.3: Matrix failure envelopes for E-glass/913 lamina subjected to in-plane transverse compression and shear loading.

Once the fracture plane angle $\alpha$ is initialized for a certain integration point, a transformation is applied to the displacement jump in that point in the local reference frame, similar as done in 2D (Chapter 3) by the rotation matrix $\mathbf{R}_{\alpha}$ :

$$
\mathbf{R}_{\alpha} \overline{\mathbf{j}}=\left[\begin{array}{ccc}
\cos (\alpha) & 0 & \sin (\alpha) \\
0 & 1 & 0 \\
-\sin (\alpha) & 0 & \cos (\alpha)
\end{array}\right]\left[\begin{array}{c}
j_{n} \\
j_{s} \\
j_{t}
\end{array}\right]=\left[\begin{array}{c}
j_{n}^{*} \\
j_{s}^{*} \\
j_{t}^{*}
\end{array}\right]=\overline{\mathbf{j}}^{*}
$$

In the $\left\{n^{*}, s^{*}, t^{*}\right\}$-frame, the cohesive law (1.1) is applied and the obtained traction vector $\overline{\mathbf{t}}^{*}$ and its linearization with respect to the displacement jump $\overline{\mathbf{T}}^{*}=\delta \overline{\mathbf{t}}^{*} / \delta \overline{\mathbf{j}}^{*}$ are transformed back into the local $\{n, s, t\}$ frame.

$$
\begin{aligned}
\mathbf{R}_{\alpha}^{\mathrm{T}} \overline{\mathbf{t}}^{*}=\left[\begin{array}{ccc}
\cos (\alpha) & 0 & -\sin (\alpha) \\
0 & 1 & 0 \\
\sin (\alpha) & 0 & \cos (\alpha)
\end{array}\right]\left[\begin{array}{c}
t_{n}^{*} \\
t_{s}^{*} \\
t_{t}^{*}
\end{array}\right]=\left[\begin{array}{c}
t_{n} \\
t_{s} \\
t_{t}
\end{array}\right]=\overline{\mathbf{t}} \\
\mathbf{R}_{\alpha}^{\mathrm{T}} \overline{\mathbf{T}}^{*} \mathbf{R}_{\alpha}=\left[\begin{array}{ccc}
\cos (\alpha) & 0 & -\sin (\alpha) \\
0 & 1 & 0 \\
\sin (\alpha) & 0 & \cos (\alpha)
\end{array}\right] \overline{\mathbf{T}}^{*}\left[\begin{array}{ccc}
\cos (\alpha) & 0 & \sin (\alpha) \\
0 & 1 & 0 \\
-\sin (\alpha) & 0 & \cos (\alpha)
\end{array}\right]=\overline{\mathbf{T}}
\end{aligned}
$$


$\overline{\mathbf{t}}$ and $\overline{\mathbf{T}}$ are then transformed in the global coordinates with (1.13) and (1.14). Note that the transformation from the displacement jump in the global $\{x, y, z\}$ coordinates to the inclined fracture plane $\left\{n^{*}, s^{*}, t^{*}\right\}$ is basically described by two separate rotations: A rotation $\theta$ over the $z$-axis and a rotation $\alpha$ over the $s$-axis:

$$
\mathbf{R}_{\alpha} \mathbf{R}_{\theta} \mathbf{j}=\overline{\mathbf{j}}^{*}
$$

Since the element force vector and the element stiffness matrix in (1.15), (1.16) and (4.14) are defined by an integral over the fracture plane, the difference in length of the fracture surface in $s$ and $s^{*}$-direction of the fracture surface has to be taken into account by a length-scale factor.

$$
\begin{aligned}
& \mathbf{f}_{A}^{\mathrm{int}}=\int_{\Omega_{A}} \mathbf{B}^{\mathrm{T}} \boldsymbol{\sigma} d \Omega+\frac{1}{\cos (\alpha)} \int_{\Gamma_{c}} \mathbf{N t} d \Gamma \\
& \mathbf{f}_{B}^{\mathrm{int}}=\int_{\Omega_{B}} \mathbf{B}^{\mathrm{T}} \boldsymbol{\sigma} d \Omega-\frac{1}{\cos (\alpha)} \int_{\Gamma_{c}} \mathbf{N t} d \Gamma \\
& \mathbf{K}=\left[\begin{array}{cc}
\mathbf{K}_{A} & \mathbf{0} \\
\mathbf{0} & \mathbf{K}_{B}
\end{array}\right]+\frac{1}{\cos (\alpha)}\left[\begin{array}{cc}
\mathbf{K}_{\mathbf{j}} & -\mathbf{K}_{\mathbf{j}} \\
-\mathbf{K}_{\mathbf{j}} & \mathbf{K}_{\mathbf{j}}
\end{array}\right]
\end{aligned}
$$

\subsection{Laminate Analysis}

The effect of the transformation in the constitutive law of cohesive segments in a 3D model is assessed with the analysis of several $[0 / \bar{\theta}]_{s}$ laminates with different fiber directions $\theta$ in the middle ply. The model is verified for $\theta=90^{\circ}$ with the transformed interface model from Chapter 3 and a mesh refinement study is done in section 4.2.1. The influence of varying fiber directions is discussed in section 4.2 .2 and the influence of different fracture plane angles is discussed in section 4.2.3.
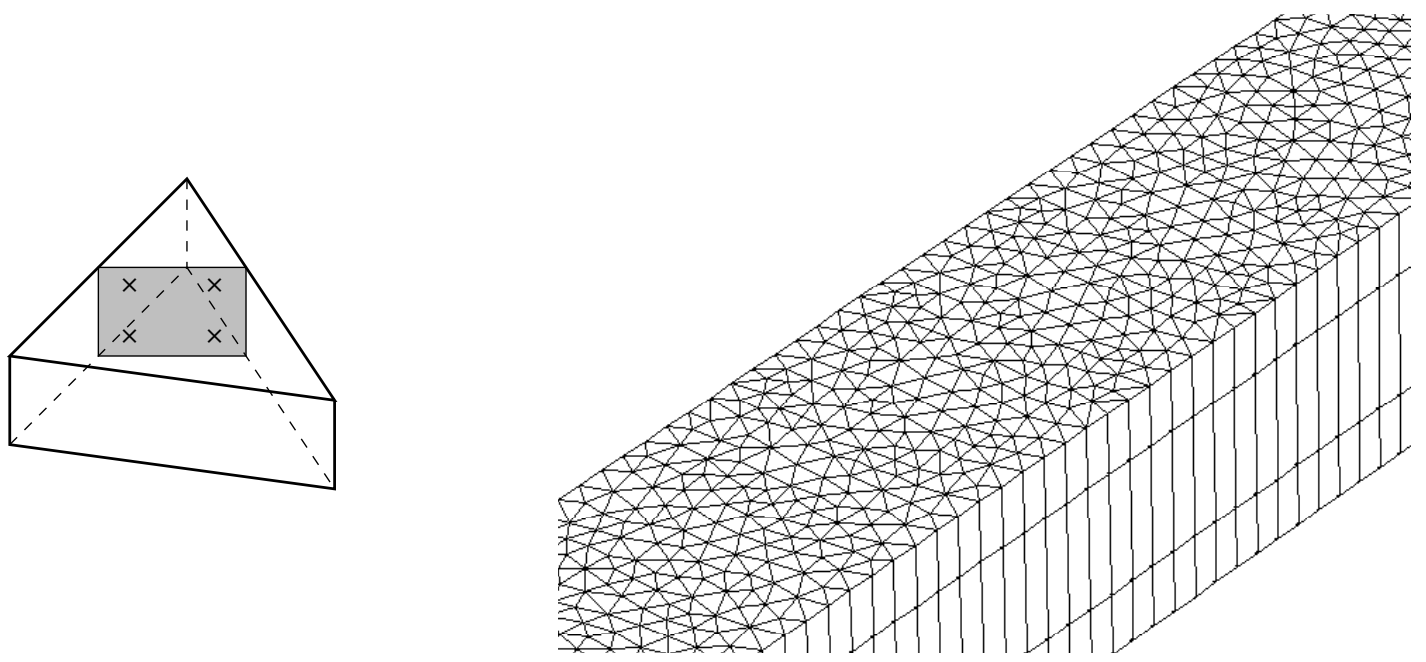

Figure 4.4: 6-noded wedge element with $2 \times 2$ Gauss integration in the cohesive segment (left) as used in the 3D laminate mesh (right).

When a $[0 / \overline{90}]_{s}$ laminate in 3D is considered with a mesh as shown in Figure 4.4, a similar failure mechanism is obtained as described in Chapter 3. The orientation of the fibers in the outer plies is in the load direction while the fibers in the middle ply are placed under an angle $\theta=90^{\circ}$ with the load direction. Therefore, the material of the outer plies is anisotropic in the analysis plane with the material properties from Table 2.1. The phantom node method is used for inter-fiber fracture, while horizontal interface elements account for delamination. The fracture plane $\alpha$ of the matrix crack is computed per integration point whenever a new crack segment is introduced. Using a damage based cohesive law for the interlaminar interface elements, snapback behavior is obtained for the transformed 3D model. Initially, the model acts linearly. As the compressive stress in the plies increases, the failure criterion in the middle ply is reached and a cohesive segment 
is introduced. Within a few iterations, the complete crack is created. The cohesive segments are damaged and the system becomes nonlinear. A wedge-shaped failure mechanism is prevented by the outer plies. This results in an increasing tensile stress in the interlaminar interface elements. When the failure criteria in these elements is met, they start to be damaged and delamination can be observed. When total damage occurs in the interlaminar interface elements near the crack, the outer plies can no longer deal with the outward pushing force and the failure load is reached. Post-peak, delamination continues.

\subsubsection{Mesh Refinement}

The transformed 3D model is verified with the 2D transformed interface model from Chapter 3 and mesh refinement is applied. If a plane stress condition is assumed in the $2 \mathrm{D}$ model, both models initially have the same stiffness. The failure load differs $1.52 \%$ if 2610 elements are used in the 3D model and $0.62 \%$ if 10412 elements are used. The load-displacement curves are shown in Figure 4.5. With 600 elements in 2D, the amount of elements along the length of a single ply is 200 . That is the same as in the fine 3D mesh and twice as much as in the course $3 \mathrm{D}$ mesh.

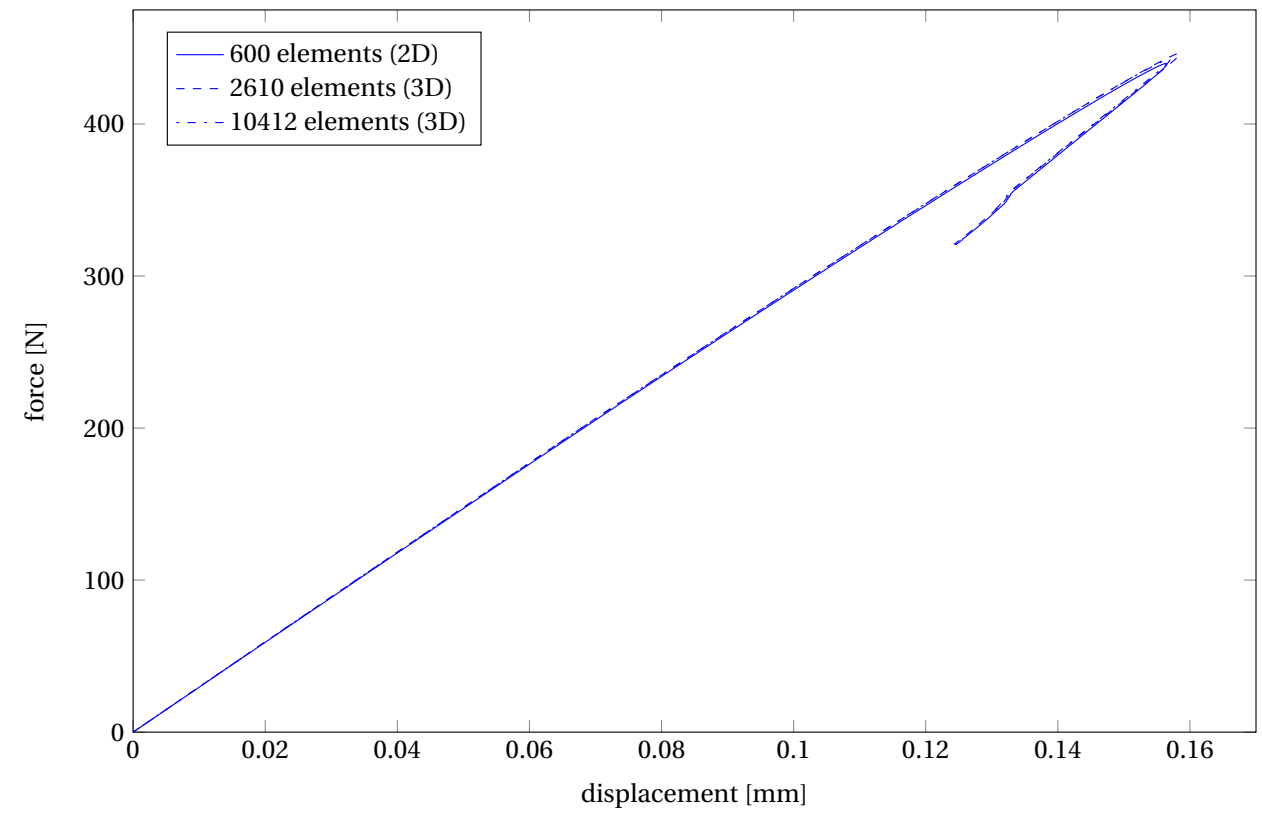

Figure 4.5: Load-displacement curves for 2D and 3D model. $L=10 \mathrm{~mm}, t_{\text {mid }}=2 t_{\text {outer }}=0.5 \mathrm{~mm}$. Material: E-glass $/ 913$.

\subsubsection{Fiber Direction}

Within the phantom node method, the orientation of the crack path $\theta$ is initialized per new cohesive segment according to the fiber orientation $\theta$. A top view of the longitudinal displacement $d x$ of the middle plies for three laminates $[0 / \overline{90}]_{s},[0 / \overline{50}]_{s}$ and $[0 / \overline{30}]_{s}$ in Figure 4.6 shows that the varying fracture plane $\alpha$ that is initialized at the same time, does not influence the direction of the crack propagation.

With different fiber directions, diferent $\sigma_{22} / \tau_{21}$-ratios are obtained, resulting in different failure modes. For the $[0 / \overline{90}]_{s}$ laminate, mode C occurs with a fracture plane $\alpha_{0}=53^{\circ}$ as in the 2D situation (Figure 4.7). The shear stress $\tau_{21}$ is unequal to zero in the $[0 / \overline{50}]_{s}$ laminate, but as shown in Figure 4.8, still mode C occurs with $\alpha>0$. For the $[0 / \overline{30}]_{s}$ laminate, $\alpha=0$ and mode B occurs (Figure 4.9 ). The initialization of the fracture plane is discussed in more detail in section 4.2.3. 


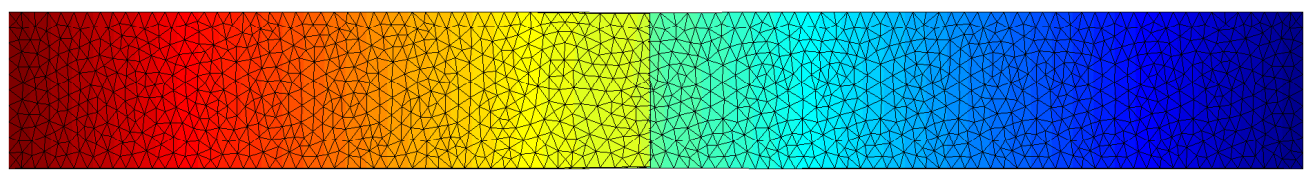

$[0 / \overline{90}]_{s}$

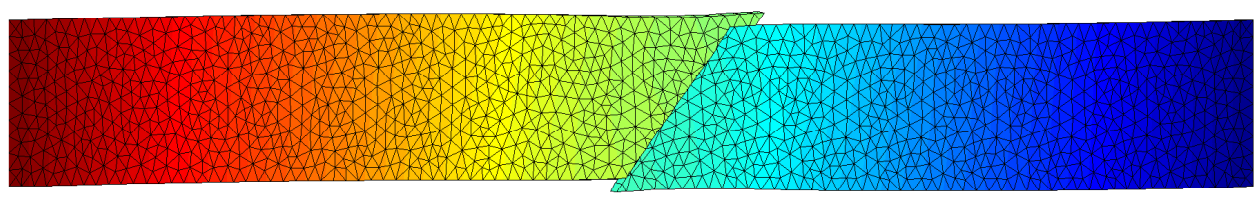

$[0 / \overline{50}]_{s}$

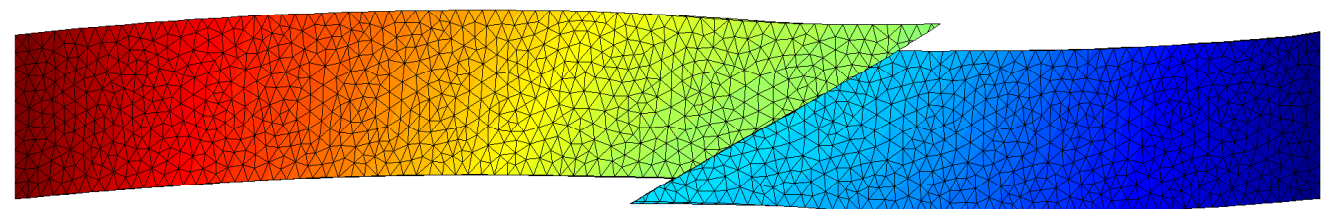

$[0 / \overline{30}]_{S}$

Figure 4.6: Displacement $d x$ of middle ply, E-glass $/ 913$ lamina at failure $($ scale factor $=10$ ) .

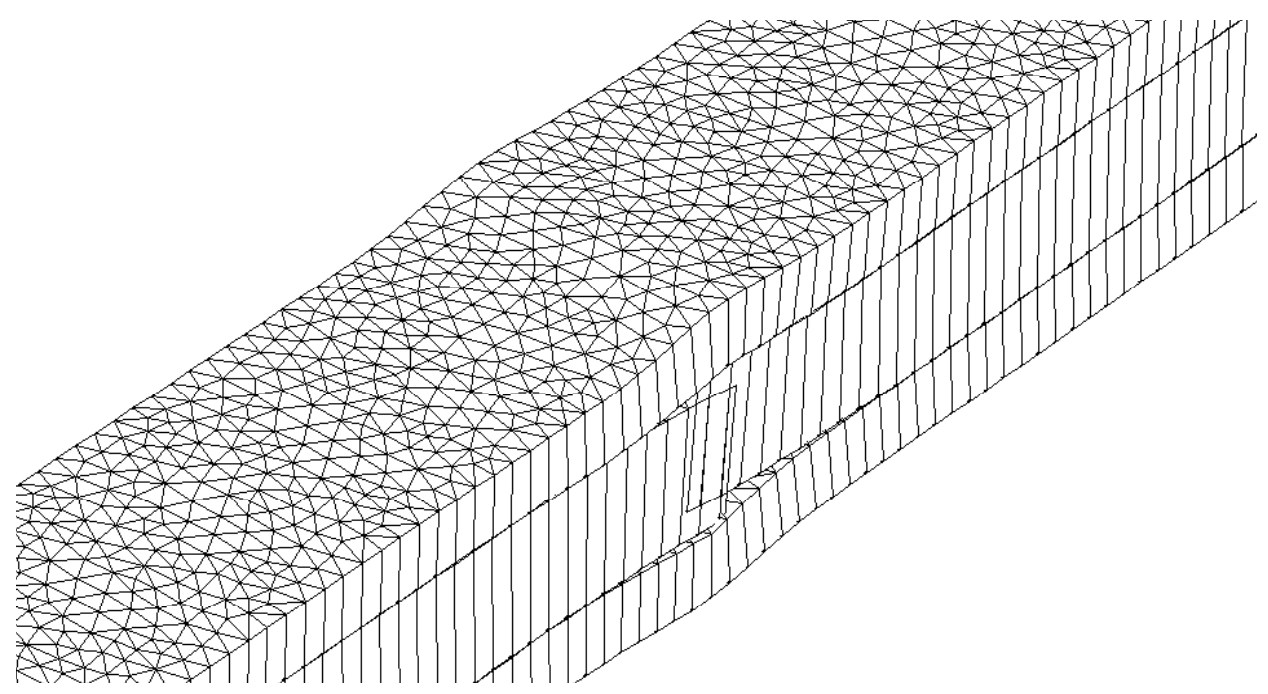

Figure 4.7: $[0 / \overline{90}]_{s}$ E-glass $/ 913$ laminate. $\alpha=\alpha_{0}=53^{\circ}($ scale factor $=10)$. 


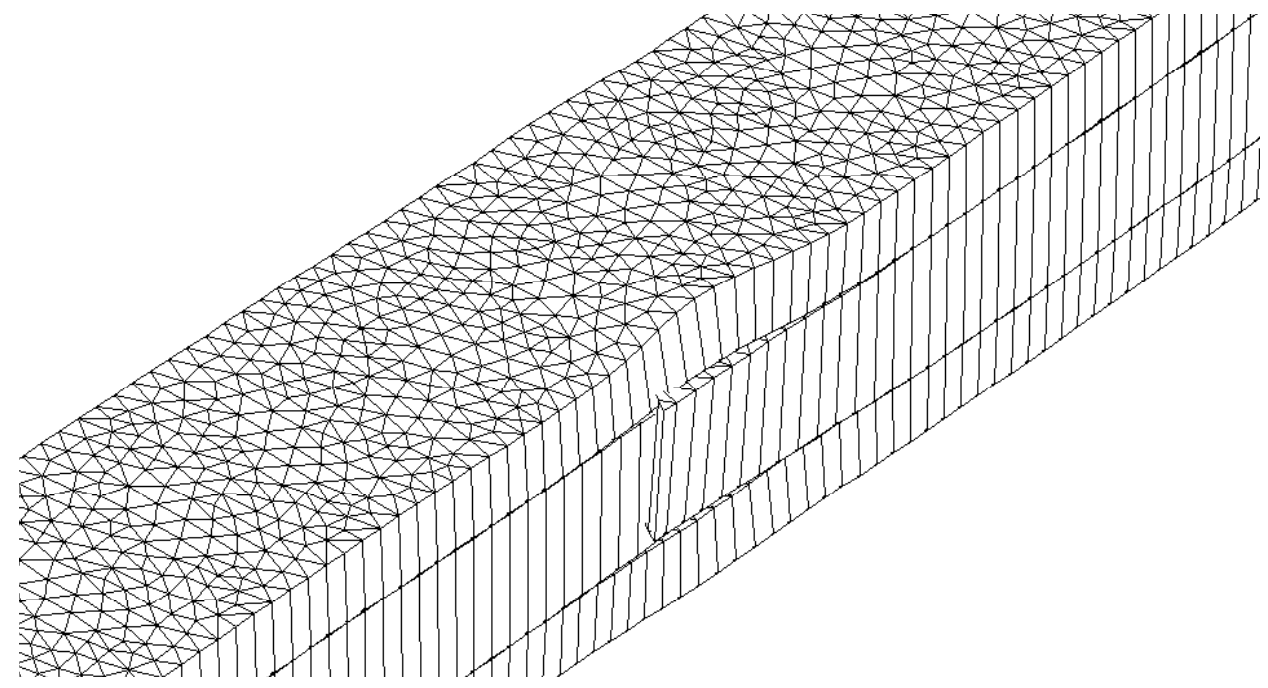

Figure 4.8: $[0 / \overline{50}]_{s}$ E-glass/913 laminate. $\alpha \approx 45^{\circ}$ (scale factor $=10$ ).

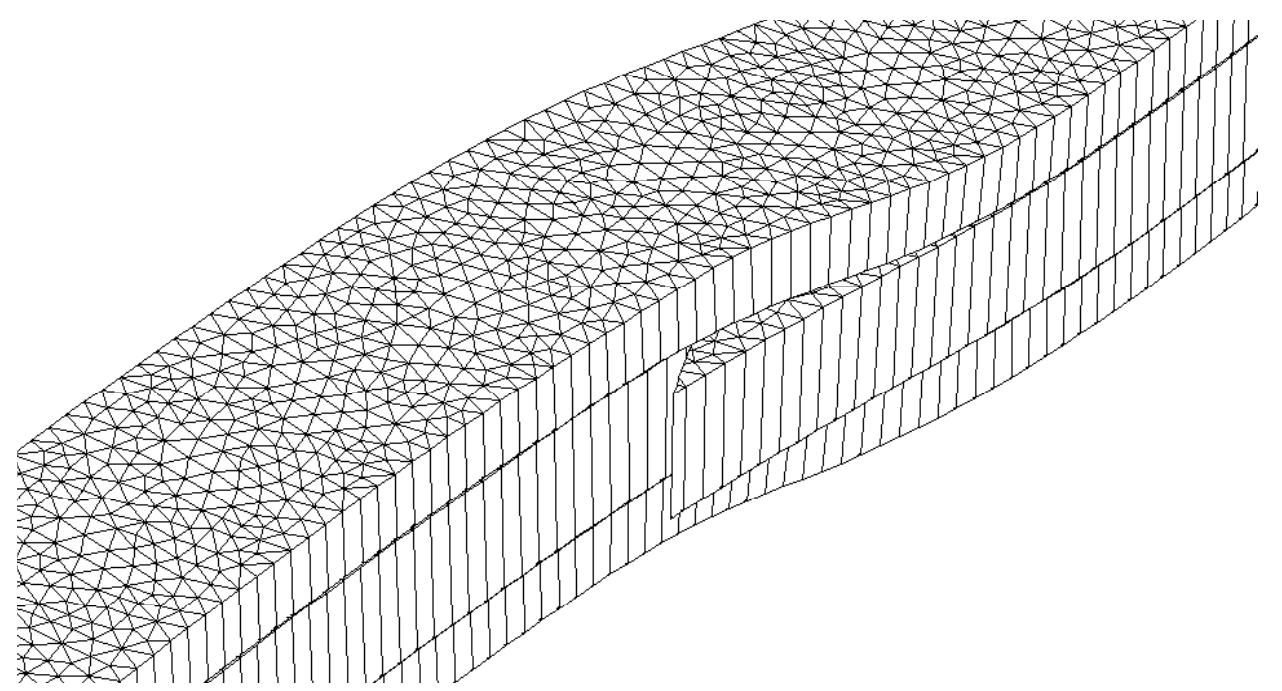

Figure 4.9: $[0 / \overline{30}]_{s}$ E-glass $/ 913$ laminate. $\alpha=0^{o}($ scale factor $=10)$. 


\subsubsection{Fracture Plane Angle}

Within the 3D model, the fracture plane is initialized for every integration point of a new created cohesive segment by the phantom node method. With an accuracy of 0.1 for $\alpha$, the difference of $\alpha$ for two neighboring integration points in an element as shown in Figure 4.4 happens to be smaller than 0.1. For neighboring elements, the difference can be much bigger, depending on the $\sigma_{22} / \tau_{21}$-ratio in that point and the failure criterion used. This is shown for several laminates in Figure 4.10. For relatively small $\tau_{21}$ values (mode C), the oscillation of $\alpha$ along the crack path is also small. For a relatively high $\tau_{21}$ (mode $\mathrm{B}$ ), $\alpha$ is zero for all integration points. When the $\sigma_{22} / \tau_{21}$-ratio oscillates between mode B and C (see Figure 4.3), a huge difference of $\alpha$ in neighboring elements can be observed. See for instance the peak for a $[0 / \overline{40}]_{s}$ laminate in Figure 4.10.

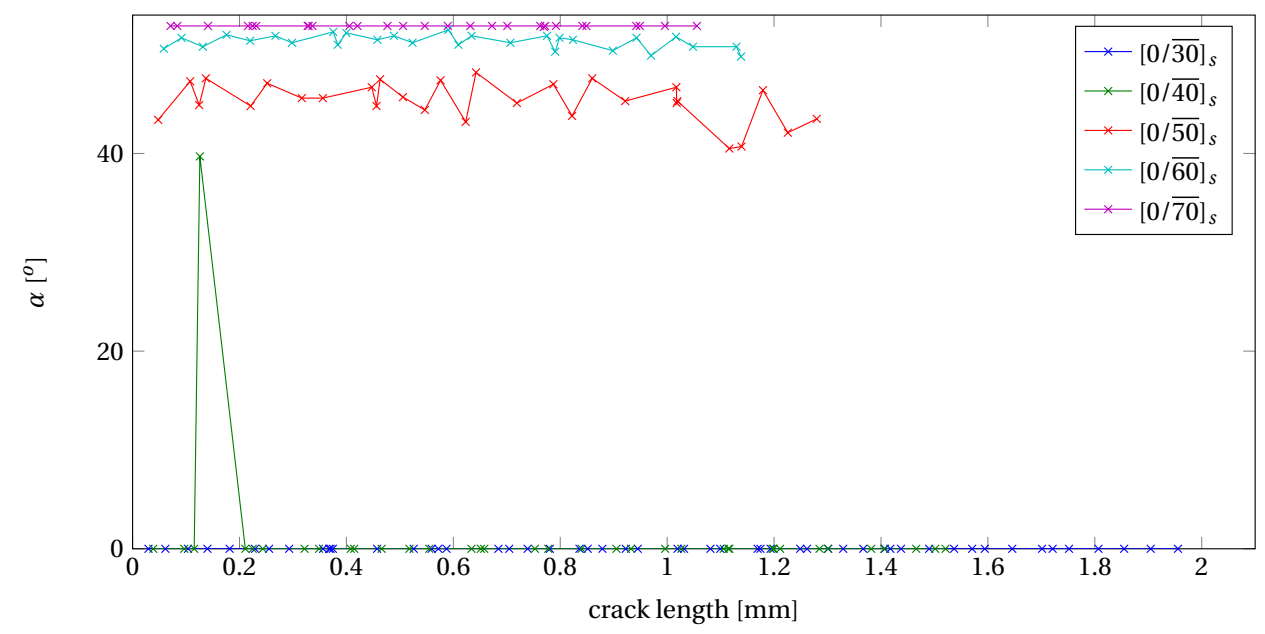

Figure 4.10: Fracture plane $\alpha$ along crack path of several $[0 / \bar{\theta}]_{s}$ laminates (2610 elements per laminate).

For a fine mesh (10412 elements), $\alpha$ is plotted per element on the crack path of a $[0 / \overline{50}]_{s}$ laminate. The oscillation does not vanish with mesh refinement, but it can also be seen that even when a mode switch occurs, it does not influence the performance of the model.

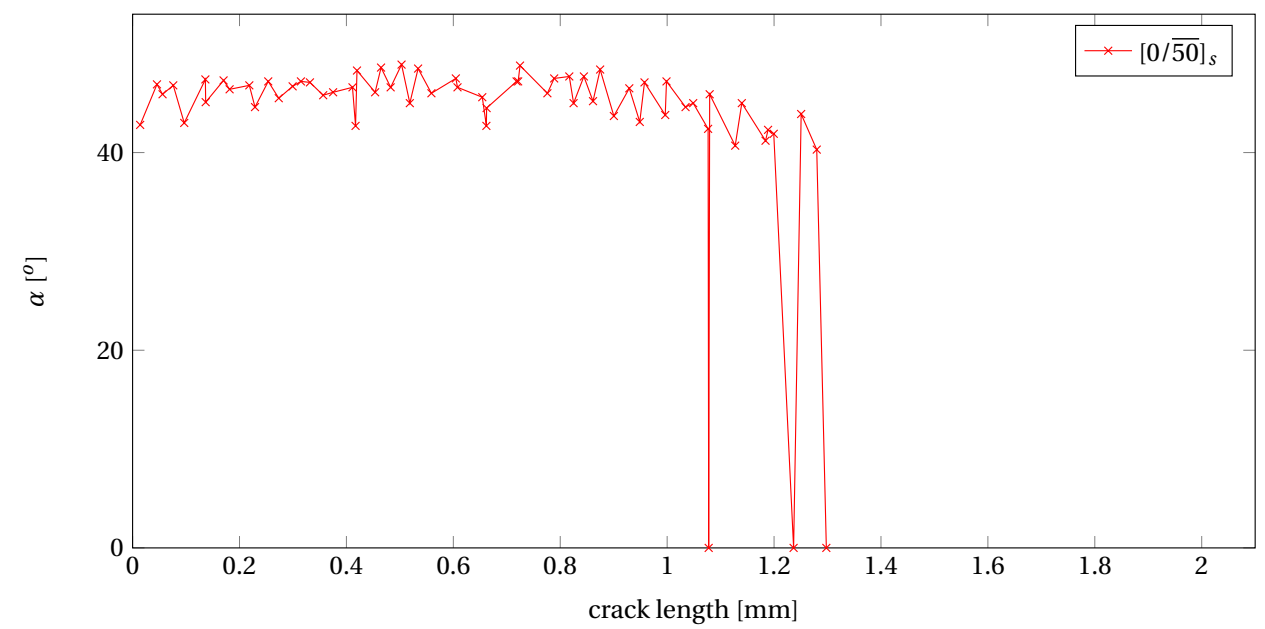

Figure 4.11: Fracture plane $\alpha$ along crack path of a $[0 / \overline{50}]_{S}$ laminate (10412 elements). 


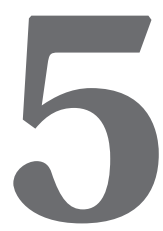

\section{CONCLUSION AND RECOMMENDATIONS}

The fracture plane of a transverse compressive loaded laminate is typically not perpendicular to the load direction. In this thesis, this compressive behavior is approximated with a vertical crack plane by applying a transformation in the cohesive law. In 2D, this is done with interface elements and a constant fracture plane angle. In 3D, the transformation is applied to the cohesive segments, created with the phantom node method where the fracture plane angle depends on the stress state in the crack tip.

Compared to a reference model with predefined crack topology by interface elements, the method of transformation in the cohesive law results in the same forces acting on the crack plane for a compressive loaded single ply. The transformation allows elements near the cracked surface to slide over a transformed axis. Therefore, interpenetration of the elements is not longer prevented and the relative displacements of the nodes along the crack are exactly the same as in the reference model.

Outer plies in a laminate initially prevent the wedge-shaped failure mechanism of a cracked middle ply. In the extreme case of relatively thick outer plies and a thin cracked middle ply, the difference in failure load of the transformation in the cohesive law is only a few percent compared to a reference model with predefined crack topology. A single layer of elements per ply is considered in this thesis, for a 3D model not to be too expensive. The linear stress distribution throughout the ply thickness that is implicitly assumed is similar for both the transformed model and the reference model. It is shown in 2D that the actual non-linear distribution for both models is different.

The transformation is basically a wrapper function around the cohesive law. Therefore, it is easily implemented in existing finite element programs. This is done for a 3D laminate failure model where the crack propagation is modeled with cohesive segments, introduced by the phantom node method. The actual fracture plane angle is dependent on the stress state in the crack tip. The angle can be computed and initialized whenever the stress state in the crack tip is evaluated. And so, the transformation can be applied to the constitutive law of the cohesive segment, even when the fracture plane angle is not known a priori. This results in a good approximation of compressive behavior in a 3D compressive loaded laminate, without the need to predefine the crack topology and without complex algorithms that deal with the crack plane in the extended finite element method.

Where possible, the method of transformation in the cohesive law is verified with a reference model with predefined interface elements for the crack topology. In 3D, it is difficult to define a reference model where the crack topology is predefined, hence the need for an approximative method. In this case, experimental results of transverse compressive loaded laminates are needed to verify the model. Unfortunately, these experimental results are not widely available yet. Nevertheless, this is the first laminate failure model that takes compressive failure into account.

In this work, one crack in the laminate is considered. In a compressive loaded laminate, typically multiple cracks occur before the ultimate failure load is reached. The actual algorithm that deals with the transformation in the cohesive law is relatively simple. This is particularly useful with many cracks in one model. It would be interesting for future work to investigate the possibilities and limitations when multiple cracks are considered. 



\section{BIBLIOGRAPHY}

[1] B. N. Cox and Q. D. Yang, In quest of virtual tests for structural composites, Science 314, 1102 (2006).

[2] F. P. van der Meer, Computational modeling of failure in composite laminates, Ph.D. thesis, Delft University of Technology (2010), available via http://repository.tudelft.nl/.

[3] A. Turon, P. P. Camanho, J. Costa, and C. G. Dávila, A damage model for the simulation of delamination in advanced composites under variable-mode loading, Mech Mater 38, 1072 (2006).

[4] F. P. van der Meer and L. J. Sluys, Continuum models for the analysis of progressive failure in composite laminates, J Compos Mater 43, 2131 (2009).

[5] N. Moës, J. Dolbow, and T. Belytschko, A finite element method for crack growth without remeshing, Int J Numer Method Eng 46, 131 (1999).

[6] A. Puck and H. Schurmann, Failure analysis of frp laminates by means of physically based phenomenological models, Composites Science and Technology 62, 1633 (2002).

[7] I. G. Barenblatt, The Mathematical Theory of Equilibrium Cracks in Brittle Fracture, Adv Appl Mech 7, 55 (1962).

[8] G. Alfano and M. A. Crisfield, Finite element interface models for the delamination analysis of laminated composites: mechanical and computational issues, Int J Numer Method Eng , 1701 (2001).

[9] F. P. van der Meer and L. J. Sluys, A phantom node formulation with mixed mode cohesive law for splitting in laminates, Int J Fract 158, 107 (2009).

[10] A. Hansbo and P. Hansbo, A finite element method for the simulation of strong and weak discontinuities in solid mechanics, Comput Method Appl Mech Eng 193, 3523 (2004).

[11] J. Mergheim, E. Kuhl, and P. Steinmann, A finite element method for the computational modelling of cohesive cracks, Int J Numer Method Eng 63, 276 (2005).

[12] J.-H. Song, P. M. A. Areias, and T. Belytschko, A method for dynamic crack and shear band propagation with phantom nodes, Int J Numer Method Eng 67, 868 (2006).

[13] S. R. Hallett and M. R. Wisnom, Numerical investigation of progressive damage and the effect of layup in notched tensile tests, Journal of Composite Materials 40, 1229 (2006).

[14] F. van der Meer and L. Sluys, Mesh-independent modeling of both distributed and discrete matrix cracking in interaction with delamination in composites, Engineering Fracture Mechanics 77, 719 (2010).

[15] C. M. Wang, C. Y. Wang, and J. N. Reddy, Exact Solutions for Buckling of Structural Members (CRC Press, USA, 2004).

[16] C. G. Davila, P. P. Camanho, and C. A. Rose, Failure criteria for frp laminates, Journal of Composite Materials 39, 323 (2005). 